\title{
ON SOME INEQUALITIES FOR SOLUTIONS OF EQUATIONS DESCRIBING THE MOTION OF A VISCOUS COMPRESSIBLE HEAT-CONDUCTING CAPILLARY FLUID BOUNDED BY A FREE SURFACE
}

Abstract. We derive inequalities for a local solution of a free boundary problem for a viscous compressible heat-conducting capillary fluid. The inequalities are crucial in proving the global existence of solutions belonging to certain anisotropic Sobolev-Slobodetskil space and close to an equilibrium state.

1. Introduction. The aim of the paper is to obtain some inequalities for a local solution of equations of motion of a viscous compressible heatconducting capillary fluid bounded by a free surface. The motion of such a fluid in a bounded domain $\Omega_{t} \subset \mathbb{R}^{3}$ (which depends on time $t \in \mathbb{R}_{+}^{1}$ ) is described by the following system with the boundary and initial conditions (see [3], [4]):

$$
\begin{array}{lr}
\varrho\left[v_{t}+(v \cdot \nabla) v\right]-\operatorname{div} \mathbb{T}(u, p)=0 & \text { in } \widetilde{\Omega}^{T}, \\
\varrho t_{t}+\operatorname{div}(\varrho v)=0 & \text { in } \widetilde{\Omega}^{T}, \\
\varrho c_{v}\left(\theta_{t}+v \cdot \nabla \theta\right)+\theta p_{\theta} \operatorname{div} v-\varkappa \Delta \theta & \\
\quad-\frac{\mu}{2} \sum_{i, j=1}^{3}\left(v_{i x_{j}}+v_{j x_{i}}\right)^{2}-(\nu-\mu)(\operatorname{div} v)^{2}=\varrho r & \text { in } \widetilde{\Omega}^{T}, \\
\mathbb{T} \bar{n}-\sigma H \bar{n}=-p_{0} \bar{n} & \text { on } \widetilde{S}^{T},
\end{array}
$$

2000 Mathematics Subject Classification: 35Q35, 35R35, 76N10.

Key words and phrases: free boundary, compressible viscous heat conducting fluid, surface tension.

Research supported by KBN grant no. 2PO3A-003-14. 
[cont.]

$$
\begin{array}{ll}
v \cdot \bar{n}=-\varphi_{t} /|\nabla \varphi| & \text { on } \widetilde{S}^{T}, \\
\partial \theta / \partial n=\bar{\theta} & \text { on } \widetilde{S}^{T}, \\
\left.\varrho\right|_{t=0}=\varrho_{0},\left.\quad v\right|_{t=0}=v_{0},\left.\quad \theta\right|_{t=0}=\theta_{0} & \text { in } \Omega,
\end{array}
$$

where $\widetilde{\Omega}^{T} \equiv \bigcup_{t \in(0, T)} \Omega_{t} \times\{t\}, \Omega_{0}=\Omega$ is an initial domain, $\widetilde{S}^{T} \equiv \bigcup_{t \in(0, T)} S_{t}$ $\times\{t\}, S_{t}=\partial \Omega_{t}, \varphi(x, t)=0$ describes $S_{t}, \bar{n}$ is the unit outward vector normal to the boundary, i.e. $\bar{n}=\nabla \varphi /|\nabla \varphi|$. Moreover, $v=v(x, t)$ is the velocity of the fluid, $\varrho=\varrho(x, t)$ the density, $\theta=\theta(x, t)$ the temperature, $r=r(x, t)$ the heat sources per unit mass, $\bar{\theta}=\bar{\theta}(x, t)$ the heat flow per unit surface, $p=p(\varrho, \theta)$ the pressure, $c_{v}=c_{v}(\varrho, \theta)$ the specific heat at constant volume, $\mu$ and $\nu$ the viscosity coefficients, $\varkappa$ the coefficient of heat conductivity, $\sigma$ the coefficient of surface tension, and $p_{0}$ the external (constant) pressure.

From the thermodynamic considerations we have

$$
\nu>\frac{1}{3} \mu>0, \quad \varkappa>0, \quad c_{v}>0, \quad \sigma>0 .
$$

Further, $\mathbb{T}=\mathbb{T}(v, p)$ denotes the stress tensor of the form

$$
\mathbb{T}(v, p)=\left\{T_{i j}\right\}_{i, j=1,2,3}=\left\{D_{i j}(v)-p \delta_{i j}\right\}_{i, j=1,2,3},
$$

where

$$
\mathbb{D}(v)=\left\{D_{i j}(v)\right\}_{i, j=1,2,3}=\left\{\mu S_{i j}(v)+(\nu-\mu) \delta_{i j} \operatorname{div} v\right\}_{i, j=1,2,3}
$$

and $\mathbb{S}(v)=\left\{v_{i x_{j}}+v_{j x_{i}}\right\}_{i, j=1,2,3}$ is the velocity deformation tensor.

Finally, we denote by $H$ the double mean curvature of $S_{t}$ which is negative for convex domains and can be expressed in the form

$$
H \bar{n}=\Delta_{S_{t}}(t) x, \quad x=\left(x_{1}, x_{2}, x_{3}\right),
$$

where $\Delta_{S_{t}}(t)$ is the Laplace-Beltrami operator on $S_{t}$. Let $S_{t}$ be determined by $x=x\left(s_{1}, s_{2}, t\right),\left(s_{1}, s_{2}\right) \in \mathbb{U} \subset \mathbb{R}^{2}$. Then we have

$$
\begin{aligned}
\Delta_{S_{t}}(t) & =g^{-1 / 2}\left(\frac{\partial}{\partial s_{\gamma}} g^{-1 / 2} \widehat{g}_{\gamma \delta} \frac{\partial}{\partial s_{\delta}}\right) \\
& =g^{-1 / 2}\left(\frac{\partial}{\partial s_{\gamma}} g^{1 / 2} g^{\gamma \delta} \frac{\partial}{\partial s_{\delta}}\right) \quad(\gamma, \delta=1,2),
\end{aligned}
$$

where the convention summation over repeated indices is assumed, $g=$ $\operatorname{det}\left\{g_{\gamma \delta}\right\}_{\gamma, \delta=1,2}, g_{\gamma \delta}=\frac{\partial x}{\partial s_{\gamma}} \cdot \frac{\partial x}{\partial s_{\delta}},\left\{g^{\gamma \delta}\right\}$ is the inverse matrix to $\left\{g_{\gamma \delta}\right\}$ and $\left\{\widehat{g}_{\gamma \delta}\right\}$ is the matrix of algebraic complements of $\left\{g_{\gamma \delta}\right\}$.

Assume that the domain $\Omega$ is given. Then by $(1.1)_{5}, \Omega_{t}=\left\{x \in \mathbb{R}^{3}: x=\right.$ $x(\xi, t), \xi \in \Omega\}$, where $x=x(\xi, t)$ is the solution of the Cauchy problem

$$
\frac{d x}{d t}=v(x, t),\left.\quad x\right|_{t=0}=\xi \in \Omega, \quad \xi=\left(\xi_{1}, \xi_{2}, \xi_{3}\right) .
$$


Integrating (1.2) we obtain

$$
x=\xi+\int_{0}^{t} u\left(\xi, t^{\prime}\right) d t^{\prime} \equiv X_{u}(\xi, t),
$$

where $u(\xi, t)=v\left(X_{u}(\xi, t), t\right)$ and $x=X_{u}(\xi, t)$ describes the relation between the Eulerian $x$ and Lagrangian $\xi$ coordinates. Moreover, by $(1.1)_{5}, S_{t}=\{x$ : $x=x(\xi, t), \xi \in S=\partial \Omega\}$.

By the continuity equation $(1.1)_{2}$ and the kinematic conditions $(1.1)_{5}$ the total mass is conserved, i.e.

$$
\int_{\Omega_{t}} \varrho(x, t) d x=\int_{\Omega} \varrho_{0}(\xi) d \xi=M .
$$

Now, assume that $p_{\varrho}>0, p_{\theta}>0$ for $\varrho, \theta \in \mathbb{R}_{+}^{1}$ and consider the equation

$$
p\left(\frac{M}{\frac{4}{3} \pi R_{e}^{3}}, \theta_{e}\right)=p_{0}+\frac{2 \sigma}{R_{e}} .
$$

We assume that there exist $R_{e}>0$ and $\theta_{e}>0$ satisfying (1.3). Then we have the following definition.

Definition 1.1. Let $r=\bar{\theta}=0$. By an equilibrium state we mean a solution $\left(v, \theta, \varrho, \Omega_{t}\right)$ of problem (1.1) such that $v=0, \theta=\theta_{e}, \varrho=\varrho_{e}$, $\Omega_{t}=\Omega_{e}$ for $t \geq 0$, where $\varrho_{e}=M /\left(\frac{4}{3} \pi R_{e}^{3}\right), \Omega_{e}$ is a ball of radius $R_{e}$, and $R_{e}>0$ and $\theta_{e}>0$ satisfy equation (1.3).

Next, we introduce

$$
\varrho_{\sigma}=\varrho-\varrho_{e}, \quad \theta_{\sigma}=\theta-\theta_{e} .
$$

Then problem (1.1) takes the form

$$
\begin{array}{ll}
\varrho\left[v_{t}+(v \cdot \nabla) v\right]-\operatorname{div} \mathbb{T}\left(v, p_{\sigma}\right)=0 & \text { in } \widetilde{\Omega}^{T}, \\
\varrho_{\sigma t}+v \cdot \nabla \varrho_{\sigma}+\varrho \operatorname{div} v=0 & \text { in } \widetilde{\Omega}^{T}, \\
\varrho c_{v}\left(\theta_{\sigma t}+v \cdot \nabla \theta_{\sigma}\right)-\varkappa \Delta \theta_{\sigma}+\theta p_{\theta} \operatorname{div} v & \\
\quad=\frac{\mu}{2} \sum_{i, j=1}^{3}\left(v_{i x_{j}}+v_{j x_{i}}\right)^{2}+(\nu-\mu)(\operatorname{div} v)^{2}+\varrho r & \text { in } \widetilde{\Omega}^{T}, \\
\mathbb{T}\left(v, p_{\sigma}\right) \bar{n}-\sigma\left(H+H_{e}\right) \bar{n}=0 & \text { on } \widetilde{S}^{T}, \\
v \cdot \bar{n}=-\varphi_{t} /|\nabla \varphi| & \text { on } \widetilde{S}^{T}, \\
\partial \theta_{\sigma} / \partial n=\bar{\theta} & \text { on } \widetilde{S}^{T} \\
\left.\varrho_{\sigma}\right|_{t=0}=\varrho_{\sigma 0}=\varrho_{0}-\varrho_{e},\left.\quad \theta_{\sigma}\right|_{t=0}=\theta_{\sigma 0}=\theta_{0}-\theta_{e}, & \text { in } \Omega, \\
\left.v\right|_{t=0}=v_{0} &
\end{array}
$$

where

$$
p_{\sigma}=p-\sigma H_{e}-p_{0}, \quad H_{e}=2 / R_{e}
$$


On the other hand we can write

$$
p_{\sigma}(\varrho, \theta)=p_{1} \varrho_{\sigma}+p_{2} \theta_{\sigma},
$$

where

$$
\begin{aligned}
p_{1}(\varrho, \theta) & =\int_{0}^{1} p_{\varrho}\left(\varrho_{e}+s\left(\varrho-\varrho_{e}\right), \theta\right) d s \\
p_{2}(\theta) & =\int_{0}^{1} p_{\theta}\left(\varrho_{e}, \theta_{e}+s\left(\theta-\theta_{e}\right)\right) d s .
\end{aligned}
$$

Problem (1.4) written in Lagrangian coordinates has the following form:

$$
\begin{array}{ll}
\eta u_{t}-\operatorname{div}_{u} \mathbb{T}_{u}\left(u, p_{\sigma}\right)=0 & \text { in } \Omega^{T}=\Omega \times(0, T), \\
\eta_{\sigma t}+\eta \operatorname{div}_{u} u=0 & \text { in } \Omega^{T}, \\
\eta c_{v} \vartheta_{\sigma t}+\vartheta p_{\vartheta} \operatorname{div}_{u} u-\varkappa \nabla_{u}^{2} \vartheta_{\sigma} & \\
\quad=\frac{\mu}{2} \sum_{i, j=1}^{3}\left(\xi_{x_{i}} \cdot \nabla_{\xi} u_{j}+\xi_{x_{j}} \cdot \nabla_{\xi} u_{i}\right)^{2} & \\
\quad-(\nu-\mu)\left(\operatorname{div}_{u} u\right)^{2}+\eta k & \text { in } \Omega^{T}, \\
\mathbb{T}_{u}\left(u, p_{\sigma}\right) \bar{n}_{u}-\sigma\left(H+H_{e}\right) \bar{n}_{u}=0 & \text { on } S^{T}, \\
\bar{n}_{u} \cdot \nabla_{u} \vartheta_{\sigma}=\bar{\vartheta} & \text { on } S^{T}, \\
\left.\eta_{\sigma}\right|_{t=0}=\varrho_{\sigma 0},\left.\quad \vartheta_{\sigma}\right|_{t=0}=\theta_{\sigma 0},\left.\quad u\right|_{t=0}=v_{0} & \text { in } \Omega,
\end{array}
$$

where $\eta(\xi, t)=\varrho\left(X_{u}(\xi, t), t\right), \vartheta(\xi, t)=\theta\left(X_{u}(\xi, t), t\right), \eta_{\sigma}=\eta-\varrho_{e}, \vartheta_{\sigma}=\vartheta-$ $\theta_{e}, k(\xi, t)=r\left(X_{u}(\xi, t), t\right), \bar{\vartheta}(\xi, t)=\bar{\theta}\left(X_{u}(\xi, t), t\right), \bar{n}_{u}(\xi, t)=\bar{n}\left(X_{u}(\xi, t), t\right)$, $\nabla_{u}=\xi_{i x} \partial_{\xi i}=\left\{\xi_{i x_{j}} \partial_{\xi_{i}}\right\}_{j=1,2,3}, \mathbb{T}_{u}(u, p)=-p I+\mathbb{D}_{u}(u), I=\left\{\delta_{i j}\right\}_{i, j=1,2,3}$,

$$
\begin{aligned}
\mathbb{D}_{u}(u) & =\left\{D_{u i j}(u)\right\}_{i, j=1,2,3} \\
& =\left\{\mu\left(\partial_{x_{i}} \xi_{k} \partial_{\xi_{k}} u_{j}+\partial_{x_{j}} \xi_{k} \partial_{\xi_{k}} u_{i}\right)+(\nu-\mu) \delta_{i j} \operatorname{div}_{u} u\right\}_{i, j=1,2,3},
\end{aligned}
$$

$\operatorname{div}_{u} u=\nabla_{u} \cdot u=\partial_{x_{i}} \xi_{k} \partial_{\xi_{k}} u_{i}, \operatorname{div}_{u} \mathbb{T}_{u}(u, p)=\left\{\partial_{x_{j}} \xi_{k} \partial_{\xi_{k}} T_{u i j}(u, p)\right\}_{i=1,2,3}$ and $\partial_{x_{i}} \xi_{k}$ are elements of the matrix $\xi_{x}$ which is inverse to the matrix $x_{\xi}=I+\int_{0}^{t} u_{\xi}\left(\xi, t^{\prime}\right) d t^{\prime}$.

In this paper we derive estimates for problem (1.1) (see Theorems 3.2 and $3.4)$ which are essential in the proof of the global-in-time existence of solutions to $(1.1)$ such that $\left(u, \vartheta_{\sigma}, \eta_{\sigma}\right) \in W_{2}^{2+\alpha, 1+\alpha / 2}\left(\Omega^{T}\right) \times W_{2}^{2+\alpha, 1+\alpha / 2}\left(\Omega^{T}\right) \times$ $W_{2}^{1+\alpha, 1 / 2+\alpha / 2}\left(\Omega^{T}\right) \cap C\left(0, T ; W_{2}^{1+\alpha}(\Omega)\right), \alpha \in(3 / 4,1)$ (see definitions in Section 2) and close to the equilibrium state. Problem (1.1) was already examined in [8], where the global existence of more regular solutions was proved. Moreover, the free boundary problem for a viscous barotropic compressible capillary fluid has been considered in [6], [7] and [10]. 
2. Notation. By $W_{2}^{k+\alpha, k / 2+\alpha / 2}\left(\Omega^{T}\right), k \in \mathbb{N} \cup\{0\}, \alpha \in(0,1)$, we denote the Sobolev-Slobodetskil space with the norm

$$
\begin{aligned}
\|u\|_{W_{2}^{k+\alpha, k / 2+\alpha / 2}\left(\Omega^{T}\right)}= & \sum_{|\beta|+2 i \leq k}\left\|\partial_{x}^{\beta} \partial_{t}^{i} u\right\|_{L_{2}\left(\Omega^{T}\right)}^{2} \\
& +\sum_{|\beta|=k} \int_{0}^{T} \int_{\Omega} \int_{\Omega} \frac{\left|\partial_{x}^{\beta} u(x, t)-\partial_{x^{\prime}}^{\beta} u\left(x^{\prime}, t\right)\right|^{2}}{\left|x-x^{\prime}\right|^{3+2 \alpha}} d x d x^{\prime} d t \\
& +\int_{\Omega} \int_{0}^{T} \int_{0}^{T} \frac{\left|\partial_{t}^{[k / 2]} u(x, t)-\partial_{t^{\prime}}^{[k / 2]} u\left(x, t^{\prime}\right)\right|^{2}}{\left|t-t^{\prime}\right|^{1+\alpha+k-2[k / 2]}} d x d t d t^{\prime},
\end{aligned}
$$

where $\partial_{x}^{\beta}=\partial_{x_{1}}^{\beta_{1}} \partial_{x_{2}}^{\beta_{2}} \partial_{x_{3}}^{\beta_{3}}, \beta=\left(\beta_{1}, \beta_{2}, \beta_{3}\right)$ is a multi-index, $|\beta|=\beta_{1}+\beta_{2}+\beta_{3}$. Similarly we can define the norms in $W_{2}^{k+\alpha}(\Omega)$ and $W_{2}^{k+\alpha, k / \alpha+\alpha / 2}\left(S^{T}\right)$.

Moreover, we shall use the notation:

$$
\begin{aligned}
& \|u\|_{W_{2}^{k+\alpha, k / 2+\alpha / 2}\left(\Omega^{T}\right)}=\|u\|_{k+\alpha, \Omega^{T}} ; \\
& \|u\|_{W_{2}^{k+\alpha}(Q)}=\|u\|_{k+\alpha, Q}, \quad Q \in\left\{\Omega, S, S^{1}\right\}\left(S^{1}\right. \text { is the unit sphere); } \\
& \|u\|_{L_{p}(Q)}=|u|_{p, Q}, \quad p \in[1, \infty], Q \in\{\Omega, S\} ; \\
& \|u\|_{L_{2}(Q)}=\|u\|_{0, Q}, \quad Q \in\left\{\Omega, S, \Omega^{T}, S^{T}\right\} ; \\
& \|u\|_{\Omega^{T}}^{(\alpha+2, \alpha / 2+1)}=\left[\|u\|_{\alpha+2, \Omega^{T}}^{2}+T^{-\alpha}\left(\left\|u_{t}\right\|_{0, \Omega^{T}}^{2}+\sum_{|\beta|=2}\left\|\partial_{x}^{\beta} u\right\|_{0, \Omega^{T}}^{2}\right)\right. \\
& \left.\quad+\sup _{t \leq T}\|u(\cdot, t)\|_{\alpha+1, \Omega}^{2}\right]^{1 / 2} ; \\
& \|u\|_{Q^{T}}^{(\alpha, \alpha / 2)}=\left(\|u\|_{\alpha, Q^{T}}^{2}+T^{-\alpha}\|u\|_{\left.0, Q^{T}\right)^{1 / 2}}^{2}, \quad Q \in\{\Omega, S\}\right. \\
& {[u]_{\alpha, \Omega^{T}, x}=\left(\int_{0}^{T} d t \int_{\Omega} \int_{\Omega} \frac{\left|u(x, t)-u\left(x^{\prime}, t\right)\right|^{2}}{\left|x-x^{\prime}\right|^{3+2 \alpha}} d x d x^{\prime}\right)^{1 / 2} ;} \\
& {[u]_{\alpha, \Omega^{T}, t}=\left(\int_{\Omega}^{T} d x \int_{0}^{T} \int_{0}^{T} \frac{\left|u(x, t)-u\left(x, t^{\prime}\right)\right|^{2}}{\left|t-t^{\prime}\right|^{1+2 \alpha}} d t d t^{\prime}\right)^{1 / 2} .}
\end{aligned}
$$

Next, we define the isotropic Besov spaces by introducing the norm (see [1], Sect. 18)

$$
\|u\|_{B_{p}^{l}\left(\mathbb{R}^{n}\right)}=\|u\|_{L_{p}\left(\mathbb{R}^{n}\right)}+\sum_{i=1}^{n}\left(\int_{0}^{h_{0}} d h \int_{\mathbb{R}^{n}} d x \frac{\left|\Delta_{i}^{m}(h) \partial_{x_{i}}^{k} u\right|^{p}}{h^{1+(l-k) p}}\right)^{1 / p},
$$


where $p \in[1, \infty]$,

$$
\Delta_{i}^{m}(h) f(x)=\sum_{j=0}^{m}(-1)^{m-j} c_{j m} f\left(x+j h e_{i}\right),
$$

$c_{j m}=\left(\begin{array}{c}m \\ j\end{array}\right)=m ! /(j !(m-j) !), x \in \mathbb{R}^{n}, e_{i}$ is the unit vector of the $i$ th coordinate axis, $i=1, \ldots, n$ and $m>l-k, m, k \in \mathbb{N} \cup\{0\}, l \in \mathbb{R}_{+}, l \notin \mathbb{Z}$.

It is proved in [2] (see also [1], Th. 18.2) that the Besov space norms are equivalent for all $m, k$ satisfying $m>l-k$.

Now, we define the Sobolev-Slobodetskiur spaces by intoducing the norm

$$
\|u\|_{W_{p}^{l}\left(\mathbb{R}^{n}\right)}=\|u\|_{L_{p}\left(\mathbb{R}^{n}\right)}+\sum_{i=1}^{n}\left(\int_{0}^{h_{0}} d h \int_{\mathbb{R}^{n}} d x \frac{\left|\Delta_{i}(h) \partial_{x_{i}}^{[l]} u\right|^{p}}{h^{1+p(-[l])}}\right)^{1 / p},
$$

where $\Delta_{i}(h)=\Delta_{i}^{l}(h), l \notin \mathbb{Z},[l]$ is the integer part of $l$.

By the Golovkin theorem [2] the norms of the spaces $B_{p}^{l}\left(\mathbb{R}^{n}\right)$ and $W_{p}^{l}\left(\mathbb{R}^{n}\right)$ are equivalent.

Now, we recall the following imbedding for Besov spaces (see [1], Sect. 18):

$$
\partial_{x}^{\sigma} B_{p}^{l}\left(\mathbb{R}^{n}\right) \subset B_{q}^{\varrho}\left(\mathbb{R}^{n}\right) \quad \text { for } \frac{n}{p}-\frac{n}{q}+|\sigma|+\varrho \leq l .
$$

Moreover, for

$$
\varkappa=\frac{1}{l}\left(\frac{n}{p}-\frac{n}{q}+|\sigma|+\varrho\right)<1
$$

we have the interpolation inequality

$$
\left\|\partial_{x}^{\sigma} u\right\|_{B_{q}^{\sigma}\left(\mathbb{R}^{n}\right)} \leq \varepsilon^{1-\varkappa}\|u\|_{B_{p}^{l}\left(\mathbb{R}^{n}\right)}+c \varepsilon^{-\varkappa}\|u\|_{L_{p}\left(\mathbb{R}^{n}\right)} .
$$

In the above notation $B_{p}^{l}\left(\mathbb{R}^{n}\right)$ with $l \in \mathbb{Z}_{+}$is the Sobolev space.

All the above remarks can be applied to spaces of functions defined on a bounded domain $\Omega \subset \mathbb{R}^{n}$ (which has the cone property), and by using a partition of unity we can also define spaces of traces on the boundary of $\Omega$ and formulate the corresponding trace theorems.

Next, we define

$$
\|u\|_{L_{p_{1}, p_{2}}\left(\Omega^{T}\right)}=\left(\int_{0}^{T} d t\left(\int_{\Omega}|u(x, t)|^{p_{1}} d x\right)^{p_{2} / p_{1}}\right)^{1 / p_{2}}
$$

and

$$
\|u\|_{\bar{L}_{p_{1}, p_{2}}\left(\Omega^{T}\right)}=\left(\int_{\Omega} d x\left(\int_{0}^{T}|u(x, t)|^{p_{1}} d t\right)^{p_{2} / p_{1}}\right)^{1 / p_{2}},
$$

where $p_{i} \in[1, \infty], i=1,2$. 
We have the following imbeddings (see [1], Sect. 18):

$$
\begin{aligned}
& \partial_{x}^{\alpha_{1}} \partial_{t}^{\alpha_{2}} W_{2}^{l, l / 2}\left(\Omega^{T}\right) \\
& \quad \subset \begin{cases}L_{p_{1}, p_{2}}\left(\Omega^{T}\right) & \text { if } n / 2-n / p_{1}+2 / 2-2 / p_{2}+\left|\alpha_{1}\right|+2 \alpha_{2} \leq l, \\
\bar{L}_{p_{1}, p_{2}}\left(\Omega^{T}\right) & \text { if } n / 2-n / p_{2}+2 / 2-2 / p_{1}+\left|\alpha_{1}\right|+2 \alpha_{2} \leq l, \\
L_{p}\left(0, T ; B_{q}^{\sigma}(\Omega)\right) & \text { if } n / 2-n / q+2 / 2-2 / p+\left|\alpha_{1}\right|+2 \alpha_{2}+\sigma \leq l, \\
L_{p}\left(\Omega ; B_{q}^{\sigma}(0, T)\right) & \text { if } n / 2-n / p+2 / 2-2 / q+\left|\alpha_{1}\right|+2 \alpha_{2}+2 \sigma \leq l,\end{cases}
\end{aligned}
$$

where $\Omega$ has the cone property.

Moreover, the corresponding interpolation inequalities hold.

3. Inequalities for global existence. In [9] the following local existence theorem is proved.

THEOREm 3.1. Let $S \in W_{2}^{5 / 2+\alpha}, \varrho_{0} \in W_{2}^{1+\alpha}(\Omega), v_{0} \in W_{2}^{1+\alpha}(\Omega), \theta_{0} \in$ $W_{2}^{1+\alpha}(\Omega), \alpha \in[3 / 4,1), \varrho_{0} \geq \varrho_{*}>0, c_{v} \in C^{2}\left(\mathbb{R}^{2}\right), c_{v}>0, p \in C^{3}\left(\mathbb{R}^{2}\right)$, assume that $r$ and $\bar{\theta}$ have continuous derivatives of order one and two, $r, r_{x_{k}}$ and $\bar{\theta}, \bar{\theta}_{x_{k}}$ satisfy the Hölder condition with exponent $\bar{\alpha} \geq 1 / 2$ and suppose the following compatibility conditions are satisfied:

$$
\begin{array}{ll}
\Pi_{0} \mathbb{D}\left(v_{0}\right) \bar{n}_{0}=0 & \text { on } S, \\
\bar{n}_{0} \cdot \mathbb{D}\left(v_{0}\right) \bar{n}_{0}=\bar{n}_{0} \cdot\left(p\left(\varrho_{0} \theta_{0}\right)-p_{0}\right) \bar{n}_{0}+\sigma \bar{n}_{0} \cdot \Delta_{S}(0) \xi & \text { on } S, \\
\bar{n}_{0} \cdot \nabla_{\xi} \theta_{0}=\bar{\theta}(\xi, 0) & \text { on } S .
\end{array}
$$

Then there exists $T>0$ such that there exists a unique solution of problem (1.1) such that $(u, \vartheta, \eta) \in W_{2}^{2+\alpha, 1+\alpha / 2}\left(\Omega^{T}\right) \times W_{2}^{2+\alpha, 1+\alpha / 2}\left(\Omega^{T}\right) \times$ $C\left(0, T ; W_{2}^{1+\alpha}(\Omega)\right) \cap W_{2}^{1+\alpha, 1 / 2+\alpha / 2}\left(\Omega^{T}\right)$.

In order to derive global estimates we assume the following condition: $\Omega_{t}$ is diffeomorphic to a ball, so $S_{t}$ can be described by

$$
|x|=r=R(\omega, t), \quad \omega \in S^{1},
$$

where $S_{1}$ is the unit sphere and we consider the motion near the equilibrium state (see Definition 1.1).

First we obtain an energy type inequality.

THEOREM 3.2. Assume that $(v, \varrho, \theta)$ is the local solution to problem (1.1). Assume that $\varrho^{*}=|\varrho|_{\infty, \Omega^{T}}, \theta^{*}=|\theta|_{\infty, \Omega^{T}}, \varrho_{*}=\min _{\bar{\Omega}^{T}} \varrho, \theta_{*}=\min _{\bar{\Omega}^{T}} \theta$. Assume that $\alpha \in[3 / 4,1), p_{\varrho}, p_{\theta}, c_{v}$ are positive. Then

$$
\begin{aligned}
& \frac{1}{2} \frac{d}{d t} \int_{\Omega_{t}}\left(\varrho v^{2}+\frac{p_{1}}{\varrho} \varrho_{\sigma}^{2}+\frac{\varrho c_{v} p_{2}}{\theta p_{\theta}} \theta_{\sigma}^{2}\right) d x \\
& \quad+\frac{\sigma}{2} \frac{d}{d t} \int_{S_{t}} g^{\gamma \delta} \int_{0}^{t} v_{s_{\gamma}} d t^{\prime} \cdot \int_{0}^{t} v_{s_{\delta}} d t^{\prime} d s+c_{0}\left(\|v\|_{1, \Omega_{t}}^{2}+\left\|\theta_{\sigma x}\right\|_{0, \Omega_{t}}^{2}\right)
\end{aligned}
$$




$$
\begin{aligned}
\leq & \varepsilon\left(\left\|\varrho_{\sigma}\right\|_{0, \Omega_{t}}^{2}+\left\|\theta_{\sigma}\right\|_{0, \Omega_{t}}^{2}\right) \\
& +\varepsilon_{1}\left(\|v\|_{2, \Omega_{t}}^{2}+\left\|\int_{0}^{t} v d t^{\prime}\right\|_{0, S_{t}}^{2}+\left\|H(\cdot, 0)+2 / R_{e}\right\|_{0, S^{1}}^{2}\right) \\
& +a_{1}\left(\varrho^{*}, 1 / \varrho_{*}, \theta^{*}, 1 / \theta_{*}\right)\left(\|v\|_{0, \Omega_{t}}^{2}+\|r\|_{0, \Omega_{t}}^{2}+\|\bar{\theta}\|_{0, S_{t}}^{2}\right) \\
& +a_{2}\left(\varrho^{*}, 1 / \varrho_{*}, \theta^{*}, 1 / \theta_{*}\right)\left[\left(\left\|\theta_{\sigma}\right\|_{1+\alpha, \Omega_{t}}^{2}+\left\|\varrho_{\sigma}\right\|_{1+\alpha, \Omega_{t}}^{2}\right)\left(\left\|\theta_{\sigma t}\right\|_{0, \Omega_{t}}^{2}+\left\|\varrho_{\sigma t}\right\|_{0, \Omega_{t}}^{2}\right)\right. \\
& \left.+\left(\left\|\varrho_{\sigma}\right\|_{1, \Omega_{t}}^{2}+\left\|\theta_{\sigma}\right\|_{1, \Omega_{t}}^{2}\right)\left\|\theta_{\sigma}\right\|_{1+\alpha, \Omega_{t}}^{2}+\|v\|_{1, \Omega_{t}}^{2}\left(\left\|\varrho_{\sigma}\right\|_{1+\alpha, \Omega_{t}}^{2}+\left\|\theta_{\sigma}\right\|_{1+\alpha, \Omega_{t}}^{2}\right)^{2}\right],
\end{aligned}
$$

where $\varepsilon$ and $\varepsilon_{1}$ are sufficiently small constants, and $a_{1}$ and $a_{2}$ are positive continuous functions of their arguments.

Proof. Multiplying $(1.4)_{1}$ by $v$, integrating over $\Omega_{t}$, using the equation of continuity, integrating by parts and using the boundary conditions we obtain

$$
\begin{aligned}
\frac{1}{2} \frac{d}{d t} \int_{\Omega_{t}} \varrho v^{2} d x+\int_{\Omega_{t}}|\mathbb{D}(v)|^{2} d x-\int_{\Omega_{t}} & \left.p_{1} \varrho_{\sigma}+p_{2} \theta_{\sigma}\right) \operatorname{div} v d x \\
& -\sigma \int_{S_{t}}\left(\Delta_{S_{t}} x+H_{e} \bar{n}\right) \cdot v d s=0 .
\end{aligned}
$$

Muliplying (1.4) 2 by $\frac{p_{1}}{\varrho} \varrho_{\sigma}$ and $(1.4)_{3}$ by $\frac{p_{2}}{\theta p_{\theta}} \theta_{\sigma}$, integrating the results over $\Omega_{t}$ and adding to (3.2) yields

$$
\begin{aligned}
& \frac{1}{2} \frac{d}{d t} \int_{\Omega_{t}} \varrho v^{2} d x+\int_{\Omega_{t}}\left(\varrho_{\sigma t}+v \cdot \nabla \varrho_{\sigma}\right) \frac{p_{1}}{\varrho} \varrho_{\sigma} d x+\int_{\Omega_{t}} \frac{\varrho c_{v}}{\theta p_{\theta}} p_{2} \theta_{\sigma}\left(\theta_{\sigma t}+v \cdot \nabla \theta_{\sigma}\right) d x \\
& +\int_{\Omega_{t}}|\mathbb{D}(v)|^{2} d x-\int_{\Omega_{t}} \frac{\varkappa}{\theta p_{\theta}} p_{2} \theta_{\sigma} \Delta \theta_{\sigma} d x-\sigma \int_{S_{t}}\left(\Delta_{S_{t}} x+H_{e} \bar{n}\right) \cdot v d s \\
& =\int_{\Omega_{t}} \frac{p_{2}}{\theta p_{\theta}} \theta_{\sigma}\left[\frac{\mu}{2} \sum_{i, j=1}^{3}\left(v_{i x_{j}}+v_{j x_{i}}\right)^{2}+(\nu-\mu)(\operatorname{div} v)^{2}\right] d x+\int_{\Omega_{t}} \frac{\varrho p_{2}}{\theta p_{\theta}} \theta_{\sigma} r d x .
\end{aligned}
$$

By using the equation of continuity $(1.1)_{2}$ the second term on the l.h.s. of (3.3) takes the form

$$
\frac{d}{d t} \int_{\Omega_{t}} \frac{1}{2} \frac{p_{1}}{\varrho} \varrho_{\sigma}^{2} d x+I_{1}
$$

where

$$
I_{1}=-\frac{1}{2} \int_{\Omega_{t}} \varrho_{\sigma}^{2}\left[\varrho \partial_{t}\left(\frac{p_{1}}{\varrho^{2}}\right)+\varrho v \cdot \nabla\left(\frac{p_{1}}{\varrho^{2}}\right)\right] d x .
$$


Hence

$$
\begin{aligned}
\left|I_{1}\right| \leq & a_{3}\left(\varrho^{*}, 1 / \varrho_{*}, \theta^{*}, 1 / \theta_{*}\right) \int_{\Omega_{t}}\left|\varrho_{\sigma}\right|^{2}\left[\left|\varrho_{\sigma t}\right|+\left|\theta_{\sigma t}\right|+|v|\left(\left|\varrho_{\sigma x}\right|+\left|\theta_{\sigma x}\right|\right)\right] d x \\
\leq & \varepsilon\left\|\varrho_{\sigma}\right\|_{0, \Omega_{t}}^{2}+a_{4}\left(\varrho^{*}, 1 / \varrho_{*}, \theta^{*}, 1 / \theta_{*}\right)\left[\left\|\varrho_{\sigma}\right\|_{1+\alpha, \Omega_{t}}^{2}\left(\left\|\varrho_{\sigma t}\right\|_{0, \Omega_{t}}^{2}+\left\|\theta_{\sigma t}\right\|_{0, \Omega_{t}}^{2}\right)\right. \\
& \left.+\left\|\varrho_{\sigma}\right\|_{1+\alpha, \Omega_{t}}^{2}\|v\|_{1, \Omega_{t}}^{2}\left(\left\|\varrho_{\sigma}\right\|_{1+\alpha, \Omega_{t}}^{2}+\left\|\theta_{\sigma}\right\|_{1+\alpha, \Omega_{t}}^{2}\right)\right],
\end{aligned}
$$

where $a_{3}, a_{4}$ etc. denote positive continuous functions. Similarly, the third term on the l.h.s. of (3.3) takes the form

$$
\frac{d}{d t} \int_{\Omega_{t}} \frac{1}{2} \frac{\varrho c_{v} p_{2}}{\theta p_{\theta}} \theta_{\sigma}^{2}+I_{2}
$$

where

$$
I_{2}=-\frac{1}{2} \int_{\Omega_{t}} \theta_{\sigma}^{2}\left[\varrho \partial_{t}\left(\frac{c_{v} p_{2}}{\theta p_{\theta}}\right)+\varrho v \cdot \nabla\left(\frac{c_{v} p_{2}}{\theta p_{\theta}}\right)\right] d x
$$

so

$$
\begin{aligned}
\left|I_{2}\right| \leq & a_{5}\left(\varrho^{*}, 1 / \varrho_{*}, \theta^{*}, 1 / \theta_{*}\right) \int_{\Omega_{t}} \theta_{\sigma}^{2}\left[\left|\varrho_{\sigma t}\right|+\left|\theta_{\sigma t}\right|+|v|\left(\left|\varrho_{\sigma x}\right|+\left|\theta_{\sigma x}\right|\right)\right] d x \\
\leq & \varepsilon\left\|\theta_{\sigma}\right\|_{0, \Omega_{t}}^{2}+a_{6}\left(\varrho^{*}, 1 / \varrho_{*}, \theta^{*}, 1 / \theta_{*}\right)\left[\left\|\theta_{\sigma}\right\|_{1+\alpha, \Omega_{t}}^{2}\left(\left\|\varrho_{\sigma t}\right\|_{0, \Omega_{t}}^{2}+\left\|\theta_{\sigma t}\right\|_{0, \Omega_{t}}^{2}\right)\right. \\
& \left.+\left\|\theta_{\sigma}\right\|_{1+\alpha, \Omega_{t}}^{2}\|v\|_{1, \Omega_{t}}^{2}\left(\left\|\varrho_{\sigma}\right\|_{1+\alpha, \Omega_{t}}^{2}+\left\|\theta_{\sigma}\right\|_{1+\alpha, \Omega_{t}}^{2}\right)\right] .
\end{aligned}
$$

By the boundary condition (1.4) 6 the fifth term on the 1.h.s. of (3.3) is equal to

$$
-\int_{S_{t}} \frac{\varkappa p_{2}}{\theta p_{\theta}} \theta_{\sigma} \bar{\theta} d s+\int_{\Omega_{t}} \frac{\varkappa p_{2}}{\theta p_{\theta}}\left|\nabla \theta_{\sigma}\right|^{2} d x+\int_{\Omega_{t}} \nabla\left(\frac{\varkappa p_{2}}{\theta p_{\theta}}\right) \cdot \theta_{\sigma} \nabla \theta_{\sigma} d x .
$$

Denoting the last expression in (3.6) by $I_{3}$ we obtain

$$
\begin{aligned}
\left|I_{3}\right| \leq & a_{5}\left(\varrho^{*}, 1 / \varrho_{*}, \theta^{*}, 1 / \theta_{*}\right) \int_{\Omega_{t}}\left|\theta_{\sigma}\right|\left|\theta_{\sigma x}\right|\left(\left|\varrho_{\sigma x}\right|+\left|\theta_{\sigma x}\right|\right) d x \\
\leq & \varepsilon\left\|\theta_{\sigma x}\right\|_{0, \Omega_{t}}^{2}+a_{7}\left(\varrho^{*}, 1 / \varrho_{*}, \theta^{*}, 1 / \theta_{*}, \varepsilon\right) \\
& \cdot\left(\left\|\theta_{\sigma}\right\|_{1+\alpha, \Omega_{t}}^{2}\left\|\varrho_{\sigma}\right\|_{1, \Omega_{t}}^{2}+\left\|\theta_{\sigma}\right\|_{1+\alpha, \Omega_{t}}^{2}\left\|\theta_{\sigma}\right\|_{1, \Omega_{t}}^{2}\right) .
\end{aligned}
$$

In view of the considerations from Lemma 4.1 of [10] the boundary term on the 1.h.s. of (3.3) takes the form

$$
\frac{\sigma}{2} \frac{d}{d t} \int_{S_{t}} g^{\gamma \delta} \int_{0}^{t} v_{s_{\gamma}} d t^{\prime} \cdot \int_{0}^{t} v_{s_{\delta}} d t^{\prime} d s+I_{4},
$$

where 


$$
\begin{aligned}
\left|I_{4}\right| \leq & \varepsilon_{1}\left(\left\|\int_{0}^{t} v d t^{\prime}\right\|_{0, S_{t}}^{2}+\|v\|_{1, \Omega_{t}}^{2}+\left\|H(\cdot, 0)+2 / R_{e}\right\|_{0, S^{1}}^{2}\right) \\
& +a_{8}\left\|\int_{0}^{t} v d t^{\prime}\right\|_{2, S_{t}}^{2}+\|v\|_{2, \Omega_{t}}^{2}+a_{9}\|v\|_{0, \Omega_{t}}^{2},
\end{aligned}
$$

where $\varepsilon_{1} \in(0,1)$.

Taking into account (3.3)-(3.9) we obtain estimate (3.1).

Next, we obtain estimates for $\sup _{t_{1} \leq t \leq T}\|u\|_{2+\alpha, \Omega}^{2}, \sup _{t_{1} \leq t \leq T}\left\|\vartheta_{\sigma}\right\|_{2+\alpha, \Omega}^{2}$ and $\sup _{t_{1} \leq t \leq T}\left\|\eta_{\sigma}\right\|_{1+\alpha, \Omega}^{2}$, where $\left(u, \vartheta_{\sigma}, \eta_{\sigma}\right)$ is the local solution of problem (1.6) and $t_{1}>0$. To do this we use the argument from [5] (see Theorem 6).

Let $\zeta_{\lambda} \in C^{\infty}$ be a function such that $\zeta_{\lambda}(t)=1$ for $t \geq t_{0}+\lambda\left(t_{0}>0\right.$, $\left.\lambda>0, t_{0}+\lambda<T\right), \zeta_{\lambda}(t)=0$ for $t \leq t_{0}+\lambda / 2,0 \leq \zeta_{\lambda}(t) \leq 1,\left|\dot{\zeta}_{\lambda}(t)\right| \leq$ $C / \lambda$, where $\dot{\zeta}_{\lambda}=d \zeta / d t$. Let $w_{\lambda}=w \zeta_{\lambda}$, where $w \in\left\{u, \vartheta_{\sigma}, \eta_{\sigma}, k, \bar{\vartheta}\right\}$. Then $\left(u_{\lambda}, \vartheta_{\sigma \lambda}, \eta_{\sigma \lambda}\right)$ satisfies the problem

$$
\begin{aligned}
\eta u_{\lambda t}-\mu \nabla_{u}^{2} u_{\lambda}-\nu \nabla_{u} \nabla_{u} \cdot u_{\lambda}=-p_{\eta} \nabla_{u} \eta_{\sigma \lambda}-p_{\vartheta} \nabla_{u} \vartheta_{\sigma \lambda}+\eta u \dot{\zeta}_{\lambda} & \text { in } \Omega^{T}, \\
\Pi_{0} \Pi_{u} \mathbb{D}_{u}\left(u_{\lambda}\right) \bar{n}_{u}=0 & \text { on } S^{T}, \\
\bar{n}_{0} \cdot \mathbb{D}_{u}\left(u_{\lambda}\right) \bar{n}_{u}-\sigma \bar{n}_{0} \cdot \Delta_{u}\left(t^{\prime}\right) \int_{0}^{t} u_{\lambda}\left(t^{\prime}\right) d t^{\prime} & \\
\quad=\int_{0}^{t}\left[\dot{\zeta}_{\lambda} \bar{n}_{0} \cdot \mathbb{T}_{u}\left(u, p_{\sigma}\right) \bar{n}_{u}-\sigma \bar{n}_{0} \cdot \zeta_{\lambda} \dot{\Delta}_{u}\left(t^{\prime}\right)\left(\xi+\int_{0}^{t} u\left(t^{\prime \prime}\right) d t^{\prime \prime}\right)\right. & \\
\left.\quad-\zeta_{\lambda} \partial_{t^{\prime}}\left(\frac{2}{R_{e}} \bar{n}_{0} \cdot \bar{n}_{u}\right)\right] d t^{\prime}+\left(p_{1} \eta_{\sigma \lambda}+p_{2} \vartheta_{\sigma \lambda}\right) \bar{n}_{0} \cdot \bar{n}_{u} & \\
\equiv \int_{0}^{t} B\left(t^{\prime}\right) d t^{\prime}+\left(p_{1} \eta_{\sigma \lambda}+p_{2} \vartheta_{\sigma \lambda}\right) \bar{n}_{0} \cdot \bar{n}_{u} & \\
\left.u_{\lambda}\right|_{t=0}=0 & \text { on } S^{T}, \\
\eta c_{v} \vartheta_{\sigma \lambda t}+\vartheta p_{\vartheta} \operatorname{div}_{u} u_{\lambda}-\varkappa \nabla_{u}^{2} \vartheta_{\sigma \lambda} & \text { in } \Omega, \\
=\frac{\mu}{2} \sum_{i, j=1}^{3}\left(\xi_{x_{i}} \cdot \nabla_{\xi} u_{j \lambda}+\xi_{x_{j}} \cdot \nabla_{\xi} u_{i \lambda}\right) & \\
\quad \cdot\left(\xi_{x_{i}} \cdot \nabla_{\xi} u_{j}+\xi_{x_{j}} \cdot \nabla_{\xi} u_{i}\right) & \\
\quad-(\nu-\mu) \operatorname{div}_{u} u_{\lambda} \operatorname{div}_{u} u+\eta k_{\lambda}+\eta c_{v} \vartheta_{\sigma} \dot{\zeta}_{\lambda} & \\
\bar{n}_{u} \cdot \nabla_{u} \vartheta_{\sigma \lambda}=\bar{\vartheta}_{\lambda} & \text { in } \Omega^{T}, \\
\left.\vartheta_{\sigma \lambda}\right|_{t=0}=0 & \text { on } S^{T}, \\
\eta_{\sigma \lambda t}+\eta \nabla_{u} \cdot u_{\lambda}=\eta_{\sigma} \dot{\zeta}_{\lambda} & \text { in } \Omega, \\
\left.\eta_{\sigma \lambda}\right|_{t=0}=0 & \text { in } \Omega^{T}, \\
& \text { in } \Omega,
\end{aligned}
$$


where $\Pi_{0} g=g-\bar{n}_{0}\left(\bar{n}_{0} \cdot g\right), \Pi_{u} g=g-\bar{n}_{u}\left(\bar{n}_{u} \cdot g\right)$, and $\bar{n}_{0}$ is the unit outward vector normal to $S$.

Next, we introduce the differences

$$
w^{(s)}(\xi, t)=w_{\lambda}(\xi, t)-w_{\lambda}^{\prime}(\xi, t),
$$

where $w \in\left\{u, \eta_{\sigma}, \vartheta_{\sigma}, k, \bar{\vartheta}\right\}, w_{\lambda}^{\prime}(\xi, t)=w_{\lambda}(\xi, t-s)$, and

$$
w_{*}^{(s)}(\xi, t)=w(\xi, t)-w^{\prime}(\xi, t),
$$

where $w \in\{u, \eta, \vartheta\}, w^{\prime}(\xi, t)=w(\xi, t-s)$.

Then we obtain the following problem:

$$
\begin{aligned}
\eta u_{t}^{(s)} & -\mu \nabla_{u}^{2} u^{(s)}-\nu \nabla_{u} \nabla_{u} \cdot u^{(s)} \\
= & -p_{\eta} \nabla_{u} \eta_{\sigma}^{(s)}-p_{\vartheta} \nabla_{u} \vartheta_{\sigma}^{(s)} \\
& -\eta_{*}^{(s)} u_{\lambda t}^{\prime}+\mu\left(\nabla_{u}^{2}-\nabla_{u^{\prime}}^{2}\right) u_{\lambda}^{\prime} \\
& +\nu\left(\nabla_{u} \nabla_{u}-\nabla_{u^{\prime}} \nabla_{u^{\prime}}\right) \cdot u_{\lambda}^{\prime}-p_{\eta}^{\prime}\left(\nabla_{u}-\nabla_{u^{\prime}}\right) \eta_{\sigma \lambda}^{\prime} \\
& -p_{\vartheta}^{\prime}\left(\nabla_{u}-\nabla_{u^{\prime}}\right) \vartheta_{\sigma \lambda}^{\prime}-\left(p_{\eta}-p_{\eta}^{\prime}\right) \nabla_{u^{\prime}} \eta_{\sigma \lambda}^{\prime} \\
& -\left(p_{\vartheta}-p_{\vartheta}^{\prime}\right) \nabla_{u^{\prime}} \vartheta_{\sigma \lambda}^{\prime}+\eta_{*}^{(s)} u \dot{\zeta}_{\lambda} \\
& +\eta^{\prime} u\left(\dot{\zeta}_{\lambda}-\dot{\zeta}_{\lambda}^{\prime}\right)+\eta^{\prime} u_{*}^{(s)} \dot{\zeta}_{\lambda}^{\prime} \equiv F \\
\Pi_{0} \Pi_{u} & \mathbb{D}_{u}\left(u^{(s)}\right) \bar{n}_{u} \\
= & -\Pi_{0}\left(\Pi_{u} \mathbb{D}_{u}\left(u_{\lambda}^{\prime}\right) \bar{n}_{u}-\Pi_{u^{\prime}} \mathbb{D}_{u^{\prime}}\left(u_{\lambda}^{\prime}\right) \bar{n}_{u^{\prime}}\right) \equiv \Pi_{0} G \\
\bar{n}_{0} \cdot \mathbb{D}_{u}\left(u^{(s)}\right) \bar{n}_{u}-\sigma \bar{n}_{0} \cdot \Delta_{u}(t) \int_{0}^{(s)}\left(t^{\prime}\right) d t^{\prime} & \text { in } \Omega^{T}, \\
= & p_{1}(\eta, \vartheta) \eta_{\sigma}^{(s)} \bar{n}_{0} \cdot \bar{n}_{u}+p_{2}(\eta, \vartheta) \vartheta_{\sigma}^{(s)} \bar{n}_{0} \cdot \bar{n}_{u} \\
& -\bar{n}_{0}\left(\mathbb{D}_{u}\left(u_{\lambda}^{\prime}\right) \bar{n}_{u}-\mathbb{D}_{u^{\prime}}\left(u_{\lambda}^{\prime}\right) \bar{n}_{u^{\prime}}\right) \\
& +{ }^{t} \\
& +\sigma \bar{n}_{0} \cdot \int_{0}\left(\Delta_{u}\left(t^{\prime}\right)-\Delta_{u^{\prime}}\left(t^{\prime}\right)\right) u_{\lambda}^{\prime} d t^{\prime} \\
& +p_{2}(\eta, \vartheta) \vartheta_{\sigma \lambda}^{\prime} \bar{n}_{0} \cdot\left(\bar{n}_{u}-\bar{n}_{u^{\prime}}\right) \\
& \left.+\int_{0}(\eta, \vartheta)-p_{1}\left(\eta^{\prime}, \vartheta^{\prime}\right)\right] \eta_{\sigma \lambda}^{\prime} \bar{n}_{0} \cdot \bar{n}_{u^{\prime}} \\
& +\lambda / 2=0 \\
& +H_{2}\left(t^{\prime}\right) d t^{\prime} \\
& \left.+B^{\prime}\left(t^{\prime}\right)\right) d t^{\prime}+p_{1}(\eta, \vartheta) \eta_{\sigma \lambda}^{\prime} \bar{n}_{0} \cdot\left(\bar{n}_{u}-\bar{n}_{u^{\prime}}\right)
\end{aligned}
$$




$$
\begin{aligned}
& \eta c_{v} \vartheta_{\sigma t}^{(s)}-\varkappa \nabla_{u}^{2} \vartheta_{\sigma}^{(s)}=\varkappa\left(\nabla_{u}^{2}-\nabla_{u^{\prime}}^{2}\right) \vartheta_{\sigma \lambda}^{\prime}-\eta_{*}^{(s)} c_{v} \vartheta_{\sigma \lambda t}^{\prime}-\eta^{\prime} c_{v} \eta \eta_{*}^{(s)} \vartheta_{\sigma \lambda t}^{\prime} \\
& -\eta^{\prime} c_{v \vartheta} \vartheta_{*}^{(s)} \vartheta_{\sigma \lambda t}^{\prime}+\eta_{*}^{(s)} c_{v} \vartheta_{\sigma} \dot{\zeta}_{\lambda}+\eta^{\prime} c_{v} \eta_{*}^{(s)} \vartheta_{\sigma} \dot{\zeta}_{\lambda}+\eta^{\prime} c_{v \vartheta} \vartheta_{*}^{(s)} \vartheta_{\sigma} \dot{\zeta}_{\lambda} \\
& +\eta^{\prime} c_{v}^{\prime}\left(\dot{\zeta}_{\lambda}-\dot{\zeta}_{\lambda}^{\prime}\right) \vartheta_{\sigma}+\eta^{\prime} c_{v}^{\prime} \vartheta_{*}^{(s)} \dot{\zeta}_{\lambda}^{\prime} \\
& -\vartheta p_{\vartheta} \operatorname{div} u u^{(s)}-\vartheta p_{\vartheta}\left(\nabla_{u}-\nabla_{u^{\prime}}\right) \cdot u_{\lambda}^{\prime} \\
& -\vartheta_{*}^{(s)} p_{\vartheta} \operatorname{div}_{u^{\prime}} u_{\lambda}^{\prime}-\vartheta^{\prime}\left(p_{\vartheta}-p_{\vartheta}^{\prime}\right) \operatorname{div}_{u^{\prime}} u_{\lambda}^{\prime} \\
& +\frac{\mu}{2} \sum_{i, j=1}^{3}\left[\left(\xi_{x_{i}} \cdot \nabla_{\xi} u_{j \lambda}+\xi_{x_{j}} \cdot \nabla_{\xi} u_{i \lambda}\right)\left(\xi_{x_{i}} \cdot \nabla_{\xi} u_{j}+\xi_{x_{j}} \cdot \nabla_{\xi} u_{i}\right)\right. \\
& \text { [cont.] } \\
& \left.-\left(\xi_{x_{i}}^{\prime} \cdot \nabla_{\xi} u_{j \lambda}^{\prime}+\xi_{x_{j}}^{\prime} \cdot \nabla_{\xi} u_{i \lambda}^{\prime}\right)\left(\xi_{x_{i}}^{\prime} \cdot \nabla_{\xi} u_{j}^{\prime}+\xi_{x_{j}}^{\prime} \cdot \nabla_{\xi} u_{i}^{\prime}\right)\right] \\
& -(\nu-\mu)\left(\operatorname{div}_{u} u_{\lambda} \operatorname{div}_{u} u-\operatorname{div}_{u^{\prime}} u_{\lambda}^{\prime} \operatorname{div}_{u^{\prime}} u^{\prime}\right)+\eta_{*}^{(s)} k_{\lambda}+\eta^{\prime} k^{(s)} \equiv I \quad \text { in } \Omega^{T} \text {, } \\
& \bar{n}_{u} \cdot \nabla_{u} \vartheta_{\sigma}^{(s)}=-\bar{n}_{u} \cdot\left(\nabla_{u}-\nabla_{u^{\prime}}\right) \vartheta_{\sigma \lambda}^{\prime}-\left(\bar{n}_{u}-\bar{n}_{u^{\prime}}\right) \cdot \nabla_{u^{\prime}} \vartheta_{\sigma \lambda}^{\prime}+\bar{\vartheta}^{(s)} \equiv J \quad \text { on } S^{T} \text {, } \\
& \left.\vartheta_{\sigma}^{(s)}\right|_{t=t_{0}+\lambda / 2}=0 \\
& \text { in } \Omega \text {, } \\
& \eta_{\sigma t}^{(s)}+\eta \nabla_{u} \cdot u^{(s)} \\
& =\eta\left(\nabla_{u}-\nabla_{u^{\prime}}\right) \cdot u_{\lambda}^{\prime}+\eta_{*}^{(s)} \nabla_{u^{\prime}} \cdot u_{\lambda}^{\prime}+\eta_{*}^{(s)} \dot{\zeta}_{\lambda}+\eta_{\sigma}\left(\dot{\zeta}_{\lambda}-\dot{\zeta}_{\lambda}^{\prime}\right) \quad \text { in } \Omega^{T}, \\
& \left.\eta_{\sigma t}^{(s)}\right|_{t=t_{0}+\lambda / 2}=0 \\
& \text { in } \Omega \text {. }
\end{aligned}
$$

First, we prove

LEMMA 3.3. Let the assumptions of Theorem 3.1 be satisfied and let $\alpha \in(3 / 4,1)$. Then

$$
\left(\left\|u^{(s)}\right\|_{Q_{\lambda}}^{(\alpha+2, \alpha / 2+1)}\right)^{2}+\left(\left\|\vartheta_{\sigma}^{(s)}\right\|_{Q_{\lambda}}^{(\alpha+2, \alpha / 2+1)}\right)^{2} \leq c_{1}(K) \bar{K} s^{1+\bar{\omega}_{1}},
$$

where $0<s<t_{0}, \bar{\omega}_{1}>0$ is a constant, $\bar{K}=\|u\|_{2+\alpha, \Omega^{T}}^{2}+\left\|\vartheta_{\sigma}\right\|_{2+\alpha, \Omega^{T}}^{2}$,

$$
\begin{aligned}
K= & \bar{K}+\left\|\eta_{\sigma}\right\|_{1+\alpha, \Omega^{T}}^{2} \\
& +\sup _{0 \leq t \leq T}\|u\|_{1+\alpha, \Omega}^{2}+\sup _{0 \leq t \leq T}\left\|\vartheta_{\sigma}\right\|_{1+\alpha, \Omega}^{2}+\sup _{0 \leq t \leq T}\left\|\eta_{\sigma}\right\|_{1+\alpha, \Omega}^{2}
\end{aligned}
$$

$c_{1}(K)$ is a positive nondecreasing continuous function of $K, Q_{\lambda}=\Omega \times$ $\left(t_{0}+\lambda, T\right)$, and $\lambda \in(0,1)$.

Proof. By Theorem 1.2 of [7] and Lemma 3.2 of [9] we have

$$
\begin{aligned}
\left\|u^{(s)}\right\|_{Q_{\lambda}}^{(\alpha+2, \alpha / 2+1)}+\left\|\vartheta_{\sigma}^{(s)}\right\|_{Q_{\lambda}}^{(\alpha+2, \alpha / 2+1)} & \\
\leq & c\left(\|F\|_{Q_{\lambda / 2}}^{(\alpha, \alpha / 2)}+\|I\|_{Q_{\lambda / 2}}^{(\alpha, \alpha / 2)}+\|G\|_{\alpha+1 / 2, G_{\lambda / 2}}\right. \\
& \left.\quad+\left\|H_{1}\right\|_{\alpha+1 / 2, G_{\lambda / 2}}+\|J\|_{\alpha+1 / 2, G_{\lambda / 2}}+\left\|H_{2}\right\|_{G_{\lambda / 2}}^{(\alpha-1 / 2, \alpha / 2-1 / 4)}\right) .
\end{aligned}
$$

We have to estimate the terms on the right-hand side of (3.12). 
First, we estimate $K_{1}=p_{\vartheta} \nabla_{u} \vartheta_{\sigma}^{(s)}$. We have

$$
\begin{aligned}
{\left[K_{1}\right]_{\alpha, Q_{\lambda / 2}, \xi}^{2} \leq } & c \int_{t_{0}+\lambda / 2}^{T} \iint_{\Omega} \frac{\left|\eta_{\sigma}(\xi)-\eta_{\sigma}\left(\xi^{\prime}\right)\right|^{2}\left|\vartheta_{\sigma \xi}^{(s)}(\xi)\right|^{2}}{\left|\xi-\xi^{\prime}\right|^{3+2 \alpha}} d \xi d \xi^{\prime} d t \\
& +c \int_{t_{0}+\lambda / 2}^{T} \iint_{\Omega} \frac{\left|\vartheta_{\sigma}(\xi)-\vartheta_{\sigma}\left(\xi^{\prime}\right)\right|^{2}\left|\vartheta_{\sigma \xi}^{(s)}(\xi)\right|^{2}}{\left|\xi-\xi^{\prime}\right|^{3+2 \alpha}} d \xi d \xi^{\prime} d t \\
& +c \int_{t_{0}+\lambda / 2}^{T} \iint_{\Omega} \frac{\left|\int_{0}^{t}\left(u_{\xi}-u_{\xi^{\prime}}\right) d t^{\prime}\right|^{2}\left|\vartheta_{\sigma \xi}^{(s)}(\xi)\right|^{2}}{\left|\xi-\xi^{\prime}\right|^{3+2 \alpha}} d \xi d \xi^{\prime} d t \\
& +c \int_{t_{0}+\lambda / 2}^{T} \iint_{\Omega} \frac{\left|\vartheta_{\sigma \xi}^{(s)}(\xi)-\vartheta_{\sigma \xi^{\prime}}^{(s)}\left(\xi^{\prime}\right)\right|^{2}}{\left|\xi-\xi^{\prime}\right|^{3+2 \alpha}} d \xi d \xi^{\prime} d t \equiv \sum_{i=1}^{4} J_{i} .
\end{aligned}
$$

First, we get

$$
\begin{aligned}
J_{4} & =c \int_{t_{0}+\lambda / 2}^{T}\left\|\vartheta_{\sigma \xi}^{(s)}\right\|_{\alpha, \Omega}^{2} d t \\
& \leq \varepsilon \int_{t_{0}+\lambda / 2}^{T}\left\|\vartheta_{\sigma}^{(s)}\right\|_{2+\alpha, \Omega}^{2} d t+c(\varepsilon)\left\|\vartheta_{\sigma}^{(s)}\right\|_{0, Q_{\lambda / 2}}^{2} \\
& \leq \varepsilon\left\|\vartheta_{\sigma}^{(s)}\right\|_{2+\alpha, Q_{\lambda / 2}}^{2}+c(\varepsilon) s^{1+\alpha} \sup _{0<s<t_{0}} \int_{t_{0}+\lambda / 2}^{T} \frac{\left|\vartheta_{\sigma}^{(s)}\right|_{2, \Omega}^{2}}{s^{1+\alpha}} d t \\
& \leq \varepsilon\left\|\vartheta_{\sigma}^{(s)}\right\|_{2+\alpha, Q_{\lambda / 2}}^{2}+c(\varepsilon) \bar{K} s^{1+\alpha} .
\end{aligned}
$$

Next, we have

$$
\begin{aligned}
J_{1} \leq & c \sup _{t_{0}+\lambda / 2 \leq t \leq T}\left(\int_{\Omega} \frac{\left|\eta_{\sigma}(\xi)-\eta_{\sigma}\left(\xi^{\prime}\right)\right|^{4}}{\left|\xi-\xi^{\prime}\right|^{3+4(1 / 4+\alpha)}} d \xi d \xi^{\prime}\right)^{1 / 2} \\
& \cdot \int_{t_{0}+\lambda / 2}^{T}\left(\int_{\Omega} \int_{\Omega} \frac{\left|\vartheta_{\sigma \xi}^{(s)}(\xi)\right|^{4}}{\left|\xi-\xi^{\prime}\right|^{2}} d \xi d \xi^{\prime}\right)^{1 / 2} d t \\
\leq & c \sup _{t_{0}+\lambda / 2 \leq t \leq T}\left\|\eta_{\sigma}\right\|_{1+\alpha, \Omega}^{2} \int_{t_{0}+\lambda / 2}^{T}\left\|\vartheta_{\sigma \xi}^{(s)}\right\|_{\alpha, \Omega}^{2} d t \\
\leq & \varepsilon\left\|\vartheta_{\sigma}^{(s)}\right\|_{2+\alpha, Q_{\lambda / 2}}^{2}+c(K) \bar{K} s^{1+\alpha},
\end{aligned}
$$

where we have used the imbeddings $W_{2}^{1+\alpha}(\Omega) \subset W_{4}^{1 / 4+\alpha}(\Omega), W_{2}^{\alpha}(\Omega) \subset$ $L_{4}(\Omega)$ for $\alpha \geq 3 / 4$ and we have estimated $\int_{t_{0}+\lambda / 2}^{T}\left\|\vartheta_{\sigma \xi}^{(s)}\right\|_{\alpha, \Omega}^{2} d t$ in the same way as in (3.13).

Similarly, we estimate $J_{2}$ and $J_{3}$. 
Summarizing the above considerations we get

$$
\left[K_{1}\right]_{\alpha, Q_{\lambda / 2}, \xi}^{2} \leq \varepsilon\left\|\vartheta_{\sigma}^{(s)}\right\|_{2+\alpha, Q_{\lambda / 2}}^{2}+c(K) \bar{K} s^{1+\alpha},
$$

where $c(K)$ is a positive nondecreasing continuous function.

Next, we calculate

$$
\begin{aligned}
{\left[K_{1}\right]_{\alpha / 2, Q_{\lambda / 2}, t}^{2} \leq } & c \int_{\Omega} \int_{t_{0}+\lambda / 2}^{T} \int_{t_{0}+\lambda / 2}^{T} \frac{\left|\eta_{\sigma}(t)-\eta_{\sigma}\left(t^{\prime}\right)\right|^{2}\left|\vartheta_{\sigma \xi}^{(s)}\left(t^{\prime}\right)\right|^{2}}{\left|t-t^{\prime}\right|^{1+\alpha}} d \xi d t d t^{\prime} \\
& +c \int_{\Omega} \int_{t_{0}+\lambda / 2}^{T} \int_{t_{0}+\lambda / 2}^{T} \frac{\left|\vartheta_{\sigma}(t)-\vartheta_{\sigma}\left(t^{\prime}\right)\right|^{2}\left|\vartheta_{\sigma \xi}^{(s)}\left(t^{\prime}\right)\right|^{2}}{\left|t-t^{\prime}\right|^{1+\alpha}} d \xi d t d t^{\prime} \\
& +c \int_{\Omega t_{0}+\lambda / 2} \int_{t_{0}+\lambda / 2}^{T} \frac{\left|\int_{t^{\prime}}^{t} u_{\xi} d \tau\right|^{2}\left|\vartheta_{\sigma \xi}^{(s)}\left(t^{\prime}\right)\right|^{2}}{\left|t-t^{\prime}\right|^{1+\alpha}} d \xi d t d t^{\prime} \\
& +c \int_{\Omega t_{0}+\lambda / 2}^{T} \int_{t_{0}+\lambda / 2}^{T} \frac{\left|\vartheta_{\sigma \xi}^{(s)}(t)-\vartheta_{\sigma \xi}^{(s)}\left(t^{\prime}\right)\right|^{2}}{\left|t-t^{\prime}\right|^{1+\alpha}} d \xi d t d t^{\prime} \\
= & \sum_{i=5}^{8} J_{i} .
\end{aligned}
$$

We estimate $J_{8}$ in the same way as $J_{4}$.

Next, we have

$$
\begin{aligned}
J_{5}+J_{7} & \leq c \int_{0}^{T}\left|u_{\xi}\right|_{\infty, \Omega} d t \int_{\Omega} \int_{t_{0}+\lambda / 2}^{T} \int_{t_{0}+\lambda / 2}^{T} \frac{\left|\vartheta_{\sigma}^{(s)}\left(t^{\prime}\right)\right|^{2}}{\left|t-t^{\prime}\right|^{\alpha}} d \xi d t d t^{\prime} \\
& \leq c\|u\|_{2+\alpha, \Omega^{T}}^{2} \int_{t_{0}+\lambda / 2}^{T}\left|\vartheta_{\sigma \xi}^{(s)}\right|_{2, \Omega}^{2} d t \\
& \leq \varepsilon\left\|\vartheta_{\sigma}^{(s)}\right\|_{2+\alpha, Q_{\lambda / 2}}^{2}+c(K) \bar{K} s^{1+\alpha} .
\end{aligned}
$$

Finally, we get

$$
\begin{aligned}
J_{6} & \leq c \int_{\Omega} \int_{t_{0}+\lambda / 2}^{T} \int_{t_{0}+\lambda / 2}^{T} \frac{\left|\int_{t^{\prime}}^{t} \vartheta_{\sigma \tau} d \tau\right|^{2}\left|\vartheta_{\sigma \xi}^{(s)}\left(t^{\prime}\right)\right|^{2}}{\left|t-t^{\prime}\right|^{1+\alpha}} d \xi d t d t^{\prime} \\
& \leq c \int_{t_{0}+\lambda / 2}^{T}\left|\vartheta_{\sigma \tau}\right|_{4, \Omega}^{2} d t \int_{t_{0}+\lambda / 2}^{T}\left|\vartheta_{\sigma \xi}^{(s)}\right|_{4, \Omega}^{2} d t \leq c\left\|\vartheta_{\sigma}\right\|_{\alpha, Q_{\lambda / 2}}^{2} \int_{t_{0}+\lambda / 2}^{T}\left|\vartheta_{\sigma \xi}^{(s)}\right|_{4, \Omega}^{2} d t \\
& \leq \varepsilon\left\|\vartheta_{\sigma}^{(s)}\right\|_{2+\alpha, Q_{\lambda / 2}}^{2}+c(K) \bar{K} s^{1+\alpha} .
\end{aligned}
$$


Summarizing the above considerations we get

$$
\left[K_{1}\right]_{\alpha / 2, Q_{\lambda / 2}, t}^{2} \leq \varepsilon\left\|\vartheta_{\sigma}^{(s)}\right\|_{2+\alpha, Q_{\lambda / 2}}^{2}+c(K) \bar{K} s^{1+\alpha} .
$$

Estimates (3.14) and (3.15) yield

$$
\left(\left\|p_{\vartheta} \nabla_{u} \vartheta_{\sigma}^{(s)}\right\|_{Q_{\lambda / 2}}^{(\alpha, \alpha / 2)}\right)^{2} \leq \varepsilon\left\|\vartheta_{\sigma}^{(s)}\right\|_{2+\alpha, Q_{\lambda / 2}}^{2}+c(K) \bar{K} s^{1+\alpha} .
$$

The next term we consider is $K_{2}=\eta^{\prime} c_{v \vartheta} \vartheta_{*}^{(s)} \vartheta_{\sigma \lambda t}^{\prime}$.

First, we have

$$
\begin{aligned}
{\left[K_{2}\right]_{\alpha, Q_{\lambda / 2}, \xi}^{2} \leq } & c \int_{t_{0}+\lambda / 2}^{T} \iint_{\Omega} \frac{\left|\eta_{\sigma}(\xi)-\eta_{\sigma}\left(\xi^{\prime}\right)\right|^{2}\left|\vartheta_{*}^{(s)}\right|^{2}\left|\vartheta_{\sigma \lambda t}^{\prime}\right|^{2}}{\left|\xi-\xi^{\prime}\right|^{3+2 \alpha}} d t d \xi d \xi^{\prime} \\
& +c \int_{t_{0}+\lambda / 2}^{T} \iint_{\Omega} \frac{\left.\left|\vartheta_{\sigma}(\xi)-\vartheta_{\sigma}\left(\xi^{\prime}\right)\right|^{2}\left|\vartheta_{*}^{(s)}\right|\right|^{2}\left|\vartheta_{\sigma \lambda t}^{\prime}\right|^{2}}{\left|\xi-\xi^{\prime}\right|^{3+2 \alpha}} d t d \xi d \xi^{\prime} \\
& +c \int_{t_{0}+\lambda / 2}^{T} \iint_{\Omega} \frac{\left|\vartheta_{*}^{(s)}(\xi)-\vartheta_{*}^{(s)}\left(\xi^{\prime}\right)\right|^{2}\left|\vartheta_{\sigma \lambda t}^{\prime}\right|^{2}}{\left|\xi-\xi^{\prime}\right|^{3+2 \alpha}} d t d \xi d \xi^{\prime} \\
& +c \int_{t_{0}+\lambda / 2}^{T} \iint_{\Omega} \frac{\left|\vartheta_{*}^{(s)}(\xi)\right|^{2}\left|\vartheta_{\sigma \lambda t}^{\prime}(\xi)-\vartheta_{\sigma \lambda t}^{\prime}\left(\xi^{\prime}\right)\right|^{2}}{\left|\xi-\xi^{\prime}\right|^{3+2 \alpha}} d t d \xi d \xi^{\prime} \\
\equiv & \sum_{i=9}^{12} J_{i} .
\end{aligned}
$$

We estimate

$$
\begin{gathered}
J_{9} \leq c \sup _{t_{0}+\lambda / 2 \leq T \leq T}\left|\vartheta_{*}^{(s)}\right|_{\infty, \Omega}^{2} \sup _{t_{0}+\lambda / 2 \leq t \leq T}\left(\iint_{\Omega} \frac{\left|\eta_{\sigma}(\xi)-\eta_{\sigma}\left(\xi^{\prime}\right)\right|^{4}}{\left|\xi-\xi^{\prime}\right|^{3+4(1 / 4+\alpha)}}\right)^{1 / 2} \\
\cdot \int_{t_{0}+\lambda / 2}\left(\iint_{\Omega} \frac{\left|\vartheta_{\sigma \lambda t}^{\prime}\right|^{4}}{\left|\xi-\xi^{\prime}\right|^{2}} d \xi d \xi^{\prime}\right)^{1 / 2} d t .
\end{gathered}
$$

Using the imbeddings $W_{2}^{1+\alpha}(\Omega) \subset W_{4}^{1 / 4+\alpha}(\Omega)$ and $W_{2}^{\alpha}(\Omega) \subset L_{4}(\Omega)$ (which hold for $\alpha \geq 3 / 4)$ and the interpolation inequality

$$
\sup _{t_{0}+\lambda / 2 \leq t \leq T}\left|\vartheta_{*}^{(s)}\right|_{\infty, \Omega}^{2} \leq \varepsilon_{1}^{1-\varkappa}\left\|\vartheta_{*}^{(s)}\right\|_{2+\alpha, Q_{\lambda / 2}}^{2}+c \varepsilon_{1}^{-\varkappa}\left\|\vartheta_{*}^{(s)}\right\|_{0, Q_{\lambda / 2}}^{2}
$$

(which holds for $\varkappa=5 /(4+2 \alpha)$ and $\alpha>1 / 2)$ we get

$$
\begin{aligned}
J_{9} \leq & c\left[\varepsilon_{1}^{1-\varkappa}\left\|\vartheta_{*}^{(s)}\right\|_{2+\alpha, Q_{\lambda / 2}}^{2}+c \varepsilon_{1}^{-\varkappa} s^{1+\alpha} \sup _{0<s<t_{0}} \int_{t_{0}+\lambda / 2}^{T} \frac{\left|\vartheta_{*}^{(s)}\right|_{2, \Omega}^{2}}{s^{1+\alpha}} d t\right] \\
& \cdot \sup _{t_{0}+\lambda / 2 \leq t \leq T}\left\|\eta_{\sigma}\right\|_{1+\alpha, \Omega}^{2}\left\|\vartheta_{\sigma t}\right\|_{\alpha, Q_{\lambda / 2}}^{2} \\
\leq & \varepsilon\left\|\vartheta_{*}^{(s)}\right\|_{2+\alpha, Q_{\lambda / 2}}^{2}+c(K) \bar{K} s^{1+\alpha},
\end{aligned}
$$


where we have taken $\varepsilon_{1}=\left(\varepsilon /\left(c K^{2}\right)\right)^{1 /(1-\varkappa)}$ and $c(K)$ is a positive nondecreasing continuous function of $K$.

In the same way we estimate $J_{10}$.

Next, we have

$$
\begin{gathered}
J_{11} \leq c \sup _{t_{0}+\lambda / 2 \leq t \leq T}\left(\int_{\Omega} \int_{\Omega} \frac{\left|\vartheta_{*}^{(s)}(\xi)-\vartheta_{*}^{(s)}\left(\xi^{\prime}\right)\right|^{4}}{\left|\xi-\xi^{\prime}\right|^{3+4(1 / 4+\alpha-\delta)}}\right)^{1 / 2} \\
\cdot \int_{t_{0}+\lambda / 2}^{T}\left(\iint_{\Omega} \frac{\left|\vartheta_{\sigma \lambda t}\right|^{4}}{\left|\xi-\xi^{\prime}\right|^{2+2 \delta}}\right)^{1 / 2} d t,
\end{gathered}
$$

where $\delta>0$ is a sufficiently small constant such that $2+2 \delta<3$. Using the interpolation inequality

$$
\left\|\vartheta_{*}^{(s)}\right\|_{L \infty\left(t_{0}+\lambda / 2, T ; W_{4}^{1 / 4+\alpha-\delta}(\Omega)\right)} \leq \varepsilon_{1}^{1-\varkappa}\left\|\vartheta_{*}^{(s)}\right\|_{2+\alpha, Q_{\lambda / 2}}^{2}+c \varepsilon_{1}^{-\varkappa}\left\|\vartheta_{*}^{(s)}\right\|_{0, Q_{\lambda / 2}}^{2}
$$

with $\varkappa=(2+\alpha-\delta) /(2+\alpha)$ and the imbedding $W_{2}^{\alpha}(\Omega) \subset L_{4}(\Omega)$ (both holding for $\alpha \geq 3 / 4)$ we obtain

$$
J_{11} \leq \varepsilon\left\|\vartheta_{*}^{(s)}\right\|_{2+\alpha, Q_{\lambda / 2}}^{2}+c(K) \bar{K} s^{1+\alpha}
$$

where we have set $\varepsilon_{1}=(\varepsilon /(c K))^{1 /(1-\varkappa)}$.

Finally,

$$
J_{12} \leq c \sup _{t_{0}+\lambda / 2 \leq t \leq T}\left|\vartheta_{*}^{(s)}\right|_{\infty, \Omega}^{2}\left\|\vartheta_{\sigma t}\right\|_{\alpha, Q_{\lambda / 2}}^{2} \leq \varepsilon\left\|\vartheta_{*}^{(s)}\right\|_{2+\alpha, Q_{\lambda / 2}}^{2}+c(K) \bar{K} s^{1+\alpha}
$$

Taking into account the above considerations we get

$$
\left[K_{2}\right]_{\alpha, Q_{\lambda / 2}, \xi}^{2} \leq \varepsilon\left\|\vartheta_{*}^{(s)}\right\|_{2+\alpha, Q_{\lambda / 2}}^{2}+c(K) \bar{K} s^{1+\alpha},
$$

where $c(K)$ is a positive nondecreasing continuous function of $K$.

Now, we consider

$$
\begin{aligned}
{\left[K_{2}\right]_{\alpha / 2, Q_{\lambda / 2}, t}^{2} \leq } & c \int_{\Omega} \int_{t_{0}+\lambda / 2}^{T} \int_{t_{0}+\lambda / 2}^{T} \frac{\left|\eta_{\sigma}(t)-\eta_{\sigma}\left(t^{\prime}\right)\right|^{2}\left|\vartheta_{*}^{(s)}\right|^{2}\left|\vartheta_{\sigma \lambda t}^{\prime}\right|^{2}}{\left|t-t^{\prime}\right|^{1+\alpha}} d \xi d t d t^{\prime} \\
& +c \int_{\Omega} \int_{t_{0}+\lambda / 2}^{T} \int_{t_{0}+\lambda / 2}^{T} \frac{\left|\vartheta_{\sigma}(t)-\vartheta_{\sigma}\left(t^{\prime}\right)\right|^{2}\left|\vartheta_{*}^{(s)}\right|^{2}\left|\vartheta_{\sigma \lambda t}^{\prime}\right|^{2}}{\left|t-t^{\prime}\right|^{1+\alpha}} d \xi d t d t^{\prime} \\
& +c \int_{\Omega} \int_{t_{0}+\lambda / 2}^{T} \int_{t_{0}+\lambda / 2}^{T} \frac{\left|\vartheta_{*}^{(s)}(t)-\vartheta_{*}^{(s)}\left(t^{\prime}\right)\right|^{2}\left|\vartheta_{\sigma \lambda t}^{\prime}\right|^{2}}{\left|t-t^{\prime}\right|^{1+\alpha}} d \xi d t d t^{\prime} \\
& +c \int_{\Omega}^{T} \int_{t_{0}+\lambda / 2}^{T} \int_{t_{0}+\lambda / 2}^{T} \frac{\left.\left|\vartheta_{*}^{(s)}(t)\right|\right|^{2}\left|\vartheta_{\sigma \lambda t}^{\prime}(t)-\vartheta_{\sigma \lambda t}^{\prime}\left(t^{\prime}\right)\right|^{2}}{\left|t-t^{\prime}\right|^{1+\alpha}} d \xi d t d t^{\prime} \\
\equiv & \sum_{i=13} J_{i} .
\end{aligned}
$$


First, we have

$$
\begin{aligned}
J_{13} \leq & c \sup _{t_{0}+\lambda / 2 \leq t \leq T}\left|\vartheta_{*}^{(s)}\right|_{\infty, \Omega}^{2} \int_{\Omega} \int_{t_{0}+\lambda / 2}^{T} \int_{t_{0}+\lambda / 2}^{T} \frac{\left|\int_{t^{\prime}}^{t} u_{\xi} d \tau\right|^{2}\left|\vartheta_{\sigma \lambda t}^{\prime}\right|^{2}}{\left|t-t^{\prime}\right|^{1+\alpha}} d \xi d t d t^{\prime} \\
\leq & c\left[\varepsilon_{1}^{1-\varkappa}\left\|\vartheta_{*}^{(s)}\right\|_{2+\alpha, Q_{\lambda / 2}}^{2}+c \varepsilon_{1}^{-\varkappa}\left\|\vartheta_{*}^{(s)}\right\|_{0, Q_{\lambda / 2}}^{2}\right] \\
& \quad \cdot \int_{t_{0}+\lambda / 2}^{T}\left|u_{\xi}\right|_{\infty, \Omega}^{2} d t \int_{t_{0}+\lambda / 2}^{T}\left|\vartheta_{\sigma \lambda t}^{\prime}\right|_{2, \Omega} d t \\
\leq & \varepsilon\left\|\vartheta_{*}^{(s)}\right\|_{2+\alpha, Q_{\lambda / 2}}^{2}+c(K) \bar{K} s^{1+\alpha},
\end{aligned}
$$

where $\varkappa=5 /(4+2 \alpha)$ and we have taken $\varepsilon_{1}=\left(\varepsilon /\left(c K^{2}\right)\right)^{1 /(1-\varkappa)}$.

Next, we get

$$
\begin{aligned}
J_{14} & \leq c \sup _{t_{0}+\lambda / 2 \leq t \leq T}\left|\vartheta_{*}^{(s)}\right|_{\infty, \Omega}^{2} \int_{t_{0}+\lambda / 2}^{T} \int_{t_{0}+\lambda / 2}^{T} \frac{\left|\int_{t^{\prime}}^{t} \vartheta_{\sigma \tau} d \tau\right|_{4, \Omega}^{2}\left|\vartheta_{\sigma \lambda t}^{\prime}\right|_{4, \Omega}^{2}}{\left|t-t^{\prime}\right|^{1+\alpha}} d t d t^{\prime} \\
& \leq c \sup _{t_{0}+\lambda / 2 \leq t \leq T}\left|\vartheta_{*}^{(s)}\right|_{\infty, \Omega}^{2} \int_{t_{0}+\lambda / 2}^{T}\left\|\vartheta_{\sigma t}\right\|_{\alpha, \Omega}^{2} d t \int_{t_{0}+\lambda / 2}^{T}\left\|\vartheta_{\sigma \lambda t}^{\prime}\right\|_{\alpha, \Omega}^{2} d t \\
& \leq \varepsilon\left\|\vartheta_{*}^{(s)}\right\|_{2+\alpha, Q_{\lambda / 2}}^{2}+c(K) \bar{K} s^{1+\alpha},
\end{aligned}
$$

where we have used the same interpolation inequality as before and the imbedding $W_{2}^{\alpha}(\Omega) \subset L_{4}(\Omega)$, which holds for $\alpha \geq 3 / 4$.

Now, we have

$$
\begin{aligned}
J_{15} & \leq c \int_{t_{0}+\lambda / 2}^{T} \int_{t_{0}+\lambda / 2}^{T} \frac{\left|\int_{t^{\prime}}^{t} \vartheta_{* \tau}^{(s)} d \tau\right|_{4, \Omega}^{2}\left|\vartheta_{\sigma \lambda t}^{\prime}\right|_{4, \Omega}^{2}}{\left|t-t^{\prime}\right|^{1+\alpha}} d t d t^{\prime} \\
& \leq c\left[\varepsilon_{1}^{1-\varkappa}\left\|\vartheta_{*}^{(s)}\right\|_{2+\alpha, Q_{\lambda / 2}}^{2}+c \varepsilon_{1}^{-\varkappa}\left\|\vartheta_{*}^{(s)}\right\|_{0, Q_{\lambda / 2}}^{2}\right]\left\|\vartheta_{\sigma \lambda t}^{\prime}\right\|_{\alpha, Q_{\lambda / 2}}^{2} \\
& \leq \varepsilon\left\|\vartheta_{*}^{(s)}\right\|_{2+\alpha, Q_{\lambda / 2}}^{2}+c(K) \bar{K} s^{1+\alpha},
\end{aligned}
$$

where $\varkappa=3 /(4 \alpha), \alpha>3 / 4$ and $\varepsilon_{1}=(\varepsilon /(c K))^{1 /(1-\varkappa)}$.

We estimate $J_{16}$ similarly to $J_{12}$.

Summarizing the above estimates we get

$$
\left[K_{2}\right]_{\alpha / 2, Q_{\lambda / 2}, t}^{2} \leq \varepsilon\left\|\vartheta_{*}^{(s)}\right\|_{2+\alpha, Q_{\lambda / 2}}^{2}+c(K) \bar{K} s^{1+\alpha} .
$$

Inequalities (3.17) and (3.18) yield

$$
\left(\left\|\eta^{\prime} c_{v \vartheta} \vartheta_{*}^{(s)} \vartheta_{\sigma \lambda t}^{\prime}\right\|_{Q_{\lambda / 2}}^{(\alpha, \alpha / 2)}\right)^{2} \leq \varepsilon\left\|\vartheta_{*}^{(s)}\right\|_{2+\alpha, Q_{\lambda / 2}}^{2}+c(K) \bar{K} s^{1+\alpha} .
$$


Notice now that

$$
\begin{aligned}
& \operatorname{div}_{u} u_{\lambda} \operatorname{div}_{u} u-\operatorname{div}_{u^{\prime}} u_{\lambda}^{\prime} \operatorname{div}_{u^{\prime}} u^{\prime} \\
& =\operatorname{div}_{u} u^{(s)} \operatorname{div}_{u} u+\left(\operatorname{div}_{u}-\operatorname{div}_{u^{\prime}}\right) u_{\lambda}^{\prime} \operatorname{div}_{u} u+\operatorname{div}_{u^{\prime}} u_{\lambda}^{\prime}\left(\operatorname{div}_{u}-\operatorname{div}_{u^{\prime}}\right) u \\
& \quad+\operatorname{div}_{u^{\prime}} u_{\lambda}^{\prime} \operatorname{div}_{u^{\prime}} u_{*}^{(s)} \\
& \quad \equiv \sum_{i=3}^{6} K_{i} .
\end{aligned}
$$

Consider for example $K_{4}$. We have

$$
\begin{aligned}
& {\left[K_{4}\right]_{\alpha, Q_{\lambda / 2}, \xi}^{2}} \\
& \leq c \int_{t_{0}+\lambda / 2}^{T} \iint_{\Omega} \frac{\left|\int_{t-s}^{t}\left(u_{\xi}-u_{\xi^{\prime}}\right) d \tau\right|^{2}\left|u_{\lambda \xi}^{\prime}\right|^{2}\left|u_{\xi}\right|^{2}}{\left|\xi-\xi^{\prime}\right|^{3+2 \alpha}} d t d \xi d \xi^{\prime} \\
& \quad+c \int_{t_{0}+\lambda / 2}^{T} \iint_{\Omega} \frac{\left|\int_{t-s}^{t} u_{\xi} d \tau\right|^{2}\left|u_{\lambda \xi}^{\prime}-u_{\lambda \xi^{\prime}}^{\prime}\right|^{2}\left|u_{\xi}\right|^{2}}{\left|\xi-\xi^{\prime}\right|^{3+2+\alpha}} d t d \xi d \xi^{\prime} \\
& \quad+c \int_{t_{0}+\lambda / 2}^{T} \int_{\Omega} \frac{\left|\int_{t-s}^{t} u_{\xi} d \tau\right|^{2}\left|u_{\lambda \xi}^{\prime}\right|^{2}\left|\int_{0}^{t}\left(u_{\xi}-u_{\xi^{\prime}}\right) d \tau\right|^{2}\left|u_{\xi}\right|^{2}}{\left|\xi-\xi^{\prime}\right|^{3+2 \alpha}} d t d \xi d \xi^{\prime} \\
& \quad+c \int_{t_{0}+\lambda / 2} \int_{\Omega} \frac{\left|\int_{t-s}^{t} u_{\xi} d \tau\right|^{2}\left|u_{\lambda \xi}^{\prime}\right|^{2}\left|u_{\xi}-u_{\xi^{\prime}}\right|^{2}}{\left|\xi-\xi^{\prime}\right|^{3+2 \alpha}} d t d \xi d \xi^{\prime} \\
& \equiv \sum_{i=17}^{20} J_{i} .
\end{aligned}
$$

First, we estimate

$$
\begin{aligned}
J_{17} \leq & c \int_{t_{0}+\lambda / 2}^{T}\left|u_{\lambda \xi}^{\prime}\right|_{\infty, \Omega}^{2}\left(\int_{\Omega} \int_{\Omega} \frac{\left|\int_{t-s}^{t}\left(u_{\xi}-u_{\xi^{\prime}}\right) d \tau\right|^{4}}{\left|\xi-\xi^{\prime}\right|^{3+4(1 / 4+\alpha-\delta)}} d \xi d \xi^{\prime}\right)^{1 / 2} \\
& \cdot\left(\int_{\Omega} \int_{\Omega} \frac{\left|u_{\xi}\right|^{4}}{\left|\xi-\xi^{\prime}\right|^{2+2 \delta}} d \xi d \xi^{\prime}\right)^{1 / 2} d t
\end{aligned}
$$

where $\delta>0$ is so small that $2+2 \delta<3$. Using the interpolation inequality

$$
\left\|\int_{t-s}^{t} u_{\xi} d \tau\right\|_{W_{4}^{1 / 4+\alpha-\delta}(\Omega)}^{2} \leq \varepsilon^{1-\varkappa}\left\|\int_{t-s}^{t} u d \tau\right\|_{2+\alpha, \Omega}^{2}+c \varepsilon^{-\varkappa}\left\|\int_{t-s}^{t} u d \tau\right\|_{0, \Omega}^{2}
$$

with $\varkappa=(2+\alpha-\delta) /(2+\alpha)$ and the imbedding $W_{2}^{\alpha}(\Omega) \subset L_{4}(\Omega)$ (which 
both hold for $\alpha \geq 3 / 4$ ) and taking $\varepsilon=s$ we obtain

$$
\begin{aligned}
J_{17} \leq & c \sup _{t_{0}+\lambda / 2 \leq t \leq T}\|u\|_{1+\alpha, \Omega}^{2}\|u\|_{2+\alpha, Q_{\lambda / 2}}^{2} \\
& \cdot\left(s^{2-\varkappa}\|u\|_{2+\alpha, Q_{\lambda / 2}}^{2}+c s^{-\varkappa} s^{2} \sup _{t_{0}+\lambda / 2 \leq t \leq T}\|u\|_{0, \Omega}^{2}\right) \\
\leq & c(K) \bar{K} s^{1+\omega_{1}},
\end{aligned}
$$

where $\omega_{1}>0$.

Next, we have

$$
\begin{aligned}
J_{18} \leq & c \int_{t_{0}+\lambda / 2}^{T}\left|u_{\xi}\right|_{\infty, \Omega}^{2}\left(\iint_{\Omega} \frac{\left|u_{\xi}-u_{\xi^{\prime}}\right|^{4}}{\left|\xi-\xi^{\prime}\right|^{3+4(1 / 4+\alpha)}} d \xi d \xi^{\prime}\right)^{1 / 2} \\
& \cdot\left(\int_{\Omega} \int_{\Omega} \frac{\left|\int_{t-s}^{t} u_{\xi} d \tau\right|^{4}}{\left|\xi-\xi^{\prime}\right|^{2}} d \xi d \xi^{\prime}\right)^{1 / 2} d t .
\end{aligned}
$$

Using the imbedding $\partial_{\xi}^{\sigma} W_{2}^{2+\alpha}(\Omega) \subset W_{4}^{1 / 4+\alpha}(\Omega)$ with $|\sigma|=1$ and the interpolation inequality

$$
\left|\int_{t-s}^{t} u_{\xi} d \tau\right|_{4, \Omega}^{2} \leq \varepsilon^{1-\varkappa}\left\|\int_{t-s}^{t} u d \tau\right\|_{2+\alpha, \Omega}^{2}+c \varepsilon^{-\varkappa}\left\|\int_{t-s}^{t} u d \tau\right\|_{0, \Omega}^{2}
$$

(where $\varkappa=7 /(8+4 \alpha), \varepsilon=s$ ) we obtain as before

$$
J_{18} \leq c(K) \bar{K} s^{1+\omega_{2}}
$$

where $\omega_{2}>0$. In the same way we estimate $J_{20}$.

Finally, we get

$$
\begin{aligned}
J_{19} \leq & c \int_{t_{0}+\lambda / 2}^{T}\left|\int_{t-s}^{t} u_{\xi} d \tau\right|_{\infty, \Omega}^{2}\left|u_{\lambda \xi}^{\prime}\right|_{\infty, \Omega}^{2}\left(\int_{\Omega} \int_{\Omega} \frac{\left|\int_{0}^{t}\left(u_{\xi}-u_{\xi^{\prime}}\right) d \tau\right|^{4}}{\left|\xi-\xi^{\prime}\right|^{3+4(1 / 4+\alpha)}} d \xi d \xi^{\prime}\right)^{1 / 2} \\
& \cdot\left(\int_{\Omega} \int_{\Omega} \frac{\left|u_{\xi}\right|^{4}}{\left|\xi-\xi^{\prime}\right|^{2}} d \xi d \xi^{\prime}\right)^{1 / 2} d t \\
\leq & c(K) \bar{K} s^{1+\omega_{3}}, \quad \omega_{3}>0 .
\end{aligned}
$$

Taking into account the above considerations we obtain

$$
\left[K_{4}\right]_{\alpha, Q_{\lambda / 2}, \xi}^{2} \leq c(K) \bar{K} s^{1+\omega_{4}}
$$

where $\omega_{4}>0$.

Now, consider

$$
\begin{aligned}
& {\left[K_{4}\right]_{\alpha / 2, Q_{\lambda / 2}, t}^{2}} \\
& \quad \leq c \int_{\Omega} \int_{t_{0}+\lambda / 2}^{T} \int_{t_{0}+\lambda / 2}^{T} \frac{\left|\int_{t-s}^{t} u_{\xi} d \tau-\int_{t-s-r}^{t} u_{\xi} d \tau\right|^{2}\left|u_{\lambda \xi}^{\prime}\right|^{2}\left|u_{\xi}\right|^{2}}{\left|t-t^{\prime}\right|^{1+\alpha}} d \xi d t d t^{\prime}
\end{aligned}
$$




$$
\begin{aligned}
& +c \int_{\Omega} \int_{t_{0}+\lambda / 2}^{T} \int_{t_{0}+\lambda / 2}^{T} \frac{\left|\int_{t-s}^{t} u_{\xi} d \tau\right|^{2}\left|u_{\lambda \xi}^{\prime}(t)-u_{\lambda \xi}^{\prime}\left(t^{\prime}\right)\right|^{2}\left|u_{\xi}\right|^{2}}{\left|t-t^{\prime}\right|^{1+\alpha}} d \xi d t d t^{\prime} \\
& +c \int_{\Omega} \int_{t_{0}+\lambda / 2}^{T} \int_{t_{0}+\lambda / 2}^{T} \frac{\left|\int_{t-s}^{t} u_{\xi} d \tau\right|^{2}\left|u_{\lambda \xi}^{\prime}\right|^{2}\left|u_{\xi}\right|^{2}\left|\int_{t^{\prime}}^{t} u_{\xi} d \tau\right|^{2}}{\left|t-t^{\prime}\right|^{1+\alpha}} d \xi d t d t^{\prime} \\
& +c \int_{\Omega} \int_{t_{0}+\lambda / 2}^{T} \int_{t_{0}+\lambda / 2}^{T} \frac{\left|\int_{t-s}^{t} u_{\xi} d \tau\right|^{2}\left|u_{\lambda \xi}^{\prime}\right|^{2}\left|u_{\xi}(t)-u_{\xi}\left(t^{\prime}\right)\right|^{2}}{\left|t-t^{\prime}\right|^{1+\alpha}} d \xi d t d t^{\prime} \\
& \equiv \sum_{i=21}^{24} J_{i} .
\end{aligned}
$$

We estimate

$$
\begin{aligned}
J_{21} & \leq \int_{\Omega} \int_{t_{0}+\lambda / 2}^{T} \int_{t_{0}+\lambda / 2}^{T} \frac{\left|\int_{t^{\prime}}^{t}\left(u_{\xi}(\tau)-u_{\xi}(\tau-s)\right) d \tau\right|^{2}\left|u_{\lambda \xi}^{\prime}\right|^{2}\left|u_{\xi}\right|^{2}}{\left|t-t^{\prime}\right| 1+\alpha} d \xi d t d t^{\prime} \\
& \leq c \int_{\Omega} \int_{t_{0}+\lambda / 2}^{T} \int_{0}^{T}\left|u_{\xi}(\tau)-u_{\xi}(\tau-s)\right|^{2} d \tau\left|u_{\lambda \xi}^{\prime}\right|^{2}\left|u_{\xi}\right|^{2} d \xi d t \\
& \leq c \int_{t_{0}+\lambda / 2}^{T}\left|u_{\xi}^{(s)}\right|_{4, \Omega}^{2} d t \int_{0}^{T}\left|u_{\xi}\right|_{8, \Omega}^{4} d t \\
& \leq c\|u\|_{2+\alpha, \Omega^{T}}^{4}\left(\varepsilon_{1}^{1-\varkappa}\left\|u_{*}^{(s)}\right\|_{2+\alpha, \Omega^{T}}^{2}+c \varepsilon_{1}^{-\varkappa} s^{1+\alpha} \sup _{0<s<t_{0}} \int_{t_{0}+\lambda / 2}^{T} \frac{\left|u_{*}^{(s)}\right|_{2, \Omega}^{2}}{s^{1+\alpha}} d t\right) \\
& \leq \varepsilon\left\|u_{*}^{(s)}\right\|_{2+\alpha, Q_{\lambda / 2}}^{2}+c(K) \bar{K} s^{1+\alpha},
\end{aligned}
$$

where $\varkappa=7 /(8+4 \alpha)$ and $\varepsilon_{1}=\left(\varepsilon /\left(c K^{2}\right)\right)^{1 /(1-\varkappa)}$.

Next, we have

$$
\begin{aligned}
J_{22} \leq & c \int_{t_{0}+\lambda / 2}^{T} \int_{t_{0}+\lambda / 2}^{T}\left|\int_{t-s}^{t} u_{\xi} d \tau\right|_{\infty, \Omega}^{2}\left|u_{\xi}\right|_{4, \Omega}^{2} \frac{\left|u_{\lambda \xi}^{\prime}(t)-u_{\lambda \xi}^{\prime}\left(t^{\prime}\right)\right|_{4, \Omega}^{2}}{\left|t-t^{\prime}\right|^{1+\alpha}} d t d t^{\prime} \\
\leq & c\left(\varepsilon_{1}^{1-\varkappa} s \int_{t_{0}+\lambda / 2}^{T}\|u\|_{2+\alpha, \Omega}^{2} d t+c \varepsilon_{1}^{-\varkappa} s^{2} \sup _{t_{0}+\lambda / 2 \leq t \leq T}\|u\|_{0, \Omega}^{2}\right) \\
& \cdot \sup _{t_{0}+\lambda / 2 \leq t \leq T}\left|u_{\xi}\right|_{4, \Omega}^{2} \int_{t_{0}+\lambda / 2}^{T} \int_{t_{0}+\lambda / 2}^{T} \frac{\left\|u(t)-u\left(t^{\prime}\right)\right\|_{2, \Omega}^{2}}{\left|t-t^{\prime}\right|^{1+\alpha}} d t d t^{\prime} \\
\leq & c(K) \bar{K} s^{1+\omega_{5}},
\end{aligned}
$$

where $\varkappa=5 /(4+2 \alpha), \omega_{5}>0$ and we have taken $\varepsilon_{1}=s$. 
We estimate $J_{24}$ in the same way.

Finally,

$$
\begin{aligned}
J_{23} & \leq c \int_{t_{0}+\lambda / 2}^{T}\left|\int_{t-s}^{t} u_{\xi} d \tau\right|_{\infty, \Omega}^{2}\left|u_{\lambda \xi}^{\prime}\right|_{4, \Omega}^{2}\left|u_{\xi}\right|_{4, \Omega}^{2} \int_{t^{\prime}}^{t}\left|u_{\xi}\right|_{\infty, \Omega}^{2} d \tau d t \\
& \leq c \bar{K} \int_{t_{0}+\lambda / 2}^{T}\left(\varepsilon_{1}^{1-\varkappa}\left\|\int_{t-s}^{t} u d \tau\right\|_{2+\alpha, \Omega}^{2}+c \varepsilon_{1}^{-\varkappa}\left\|\int_{t-s}^{t} u d \tau\right\|_{0, \Omega}^{2}\right)\left|u_{\xi}\right|_{4, \Omega}^{4} d t \\
& \leq c(K) \bar{K} s^{1+\omega_{6}}
\end{aligned}
$$

where $\varkappa=5 /(4+2 \alpha), \omega_{6}>0$ and we have taken $\varepsilon_{1}=s$.

By the above calculations we get

$$
\left[K_{4}\right]_{\alpha / 2, Q_{\lambda / 2}, t}^{2} \leq \varepsilon\left\|u_{*}^{(s)}\right\|_{2+\alpha, Q_{\lambda / 2}}^{2}+c(K) \bar{K} s^{1+\omega_{7}},
$$

where $\omega_{7}>0$.

Hence, (3.20) and (3.21) yield

$$
\left(\left\|\left(\operatorname{div}_{u}-\operatorname{div}_{u^{\prime}}\right) u_{\lambda}^{\prime} \operatorname{div}_{u} u\right\|_{Q_{\lambda / 2}}^{(\alpha, \alpha / 2)}\right)^{2} \leq \varepsilon\left\|u_{*}^{(s)}\right\|_{2+\alpha, Q_{\lambda / 2}}^{2}+c(K) \bar{K} s^{1+\omega_{7}},
$$

where $\omega_{7}>0$.

The other terms on the right-hand side of (3.12) are estimated exactly in the same way and we obtain for them estimates similar to (3.16), (3.19) and (3.22).

This yields the estimate

$$
\begin{aligned}
\left(\left\|u^{(s)}\right\|_{Q_{\lambda}}^{(\alpha+2, \alpha / 2+1)}\right)^{2}+\left(\left\|\vartheta_{\sigma}^{(s)}\right\|_{Q_{\lambda}}^{(\alpha+2, \alpha / 2+1)}\right)^{2} & \\
\leq & \varepsilon\left(\left\|u^{(s)}\right\|_{2+\alpha, Q_{\lambda / 2}}^{2}+\left\|u_{*}^{(s)}\right\|_{2+\alpha, Q_{\lambda / 2}}^{2}+\left\|\vartheta_{\sigma}^{(s)}\right\|_{2+\alpha, Q_{\lambda / 2}}^{2}\right. \\
& \left.+\left\|\vartheta_{*}^{(s)}\right\|_{2+\alpha, Q_{\lambda / 2}}^{2}\right)+c(K) \bar{K} s^{1+\bar{\omega}_{1}},
\end{aligned}
$$

where $\bar{\omega}_{1}>0$ is a constant. Since $u^{(s)}=u_{*}^{(s)}$ and $\vartheta_{\sigma}^{(s)}=\vartheta_{*}^{(s)}$ on $Q_{\lambda}$ inequality (3.23) yields

$$
Y(\lambda) \leq 2 \varepsilon Y(\lambda / 2)+c(K) \bar{K} s^{1+\bar{\omega}_{1}},
$$

where

$$
\begin{aligned}
Y(\lambda)= & \left(\left\|u^{(s)}\right\|_{Q_{\lambda}}^{(\alpha+2, \alpha / 2+1)}\right)^{2}+\left(\left\|u_{*}^{(s)}\right\|_{Q_{\lambda}}^{(\alpha+2, \alpha / 2+1)}\right)^{2} \\
& +\left(\left\|\vartheta_{\sigma}^{(s)}\right\|_{Q_{\lambda}}^{(\alpha+2, \alpha / 2+1)}\right)^{2}+\left(\left\|\vartheta_{*}^{(s)}\right\|_{Q_{\lambda}}^{(\alpha+2, \alpha / 2+1)}\right)^{2} .
\end{aligned}
$$

Therefore, after iteration we get

$$
Y(\lambda) \leq \frac{1}{1-2 \varepsilon} c(K) \bar{K} s^{1+\bar{\omega}_{1}}
$$

where we assume $\varepsilon<1 / 2$.

Hence estimate (3.11) holds.

Lemma 3.3 implies 
THEOREM 3.4. Let $(u, \vartheta, \eta) \in W_{2}^{2+\alpha, 1+\alpha / 2}\left(\Omega^{T}\right) \times W_{2}^{2+\alpha, 1+\alpha / 2}\left(\Omega^{T}\right) \times$ $W_{2}^{1+\alpha, 1 / 2+\alpha / 2}\left(\Omega^{T}\right) \cap C\left(0, T ; W_{2}^{1+\alpha}(\Omega)\right)(\alpha \in(3 / 4,1))$ be the local solution of problem (1.1). Then for any $0<t_{0}<T$ and $\lambda>0$ we have $u \in C\left(t_{0}+\alpha, T\right.$; $\left.W_{2}^{2+\alpha}(\Omega)\right)$ and

$$
\begin{array}{r}
\sup _{t_{0}+\lambda \leq t \leq T}\|u\|_{2+\alpha, \Omega}^{2} \leq \bar{c}_{1}(K) \bar{K}, \\
\sup _{t_{0}+\lambda \leq t \leq T}\left\|\vartheta_{\sigma}\right\|_{2+\alpha, \Omega}^{2} \leq \bar{c}_{2}(K) \bar{K},
\end{array}
$$

where $\bar{K}=\|u\|_{2+\alpha, \Omega^{T}}^{2}+\left\|\vartheta_{\sigma}\right\|_{2+\alpha, \Omega^{T}}^{2}$,

$$
\begin{aligned}
K= & \bar{K}+\sup _{0 \leq t \leq T}\|u\|_{1+\alpha, \Omega}^{2} \\
& +\sup _{0 \leq t \leq T}\left\|\vartheta_{\sigma}\right\|_{1+\alpha, \Omega}^{2}+\left\|\eta_{\sigma}\right\|_{1+\alpha, \Omega^{T}}^{2}+\sup _{0 \leq t \leq T}\left\|\eta_{\sigma}\right\|_{1+\alpha, \Omega}^{2},
\end{aligned}
$$

and $\bar{c}_{i}(K)(i=1,2)$ are positive nondecreasing continuous functions of $K$.

Proof. First, we have

$$
\begin{aligned}
& \leq \sup _{0 \leq s \leq t_{0}} \int_{t_{0}+\lambda}^{T} \frac{\left|\|u(t)\|_{2+\alpha, \Omega}-\|u(t-s)\|_{2+\alpha, \Omega}\right|^{2}}{s^{1+\bar{w}_{1}}} d t+\int_{t_{0}+\lambda}^{T}\|u(t)\|_{2+\alpha, \Omega}^{2} d t \\
& \leq \sup _{0 \leq s \leq t_{0}} \int_{t_{0}+\lambda}^{T} \frac{\left\|u^{(s)}\right\|_{2+\alpha, \Omega}^{2}}{s^{1+\bar{w}_{1}}} d t+\int_{t_{0}+\lambda}^{T}\|u(t)\|_{2+\alpha, \Omega}^{2} d t .
\end{aligned}
$$

Now, the imbedding $B_{2, \infty}^{1 / 2+\bar{w}_{1} / 2}\left(t_{0}+\lambda, T\right) \subset B_{\infty, \infty}^{\bar{w}_{1} / 2}\left(t_{0}+\lambda, T\right)$ (which means that $\|u\|_{2+\alpha, \Omega}$ is continuous on $\left.\left[t_{0}+\lambda, T\right]\right)$ and inequalities (3.28) and (3.11) give (3.25).

Estimate (3.26) can be obtained in the same way as (3.25).

This completes the proof of the theorem.

\section{References}

[1] O. V. Besov, V. P. Il'in and S. M. Nikol'skiǔ, Integral Representations of Functions and Imbedding Theorems, Nauka, Moscow, 1975 (in Russian); English transl.: Scripta Ser. in Math., Halsted Press, 1979.

[2] K. K. Golovkin, On equivalent norms for fractional spaces, Trudy Mat. Inst. Steklov. 66 (1962), 364-383 (in Russian).

[3] L. Landau and E. Lifschitz, Hydrodynamics, Nauka, Moscow, 1986 (in Russian).

[4] J. Serrin, Mathematical principles of classical fluid mechanics, in: Handbuch der Physik, Bd. VIII/1, Springer, Berlin, 1959.

[5] V. A. Solonnikov, On an unsteady flow of a finite mass of a liquid bounded by a free surface, Zap. Nauchn. Sem. LOMI 152 (1986), 137-157 (in Russian); English transl.: J. Soviet Math. 40 (1988), 672-686. 
[6] V. A. Solonnikov and A. Tani, Evolution free boundary problem for equations of motion of viscous compressible barotropic liquid, preprint, Paderborn University.

[7] -, - Free boundary problem for a viscous compressible flow with a surface tension, in: Constantin Carathéodory: An International Tribute, T. M. Rassias (ed.), World Sci., 1991, 1270-1303.

[8] E. Zadrzyńska, On nonstationary motion of a fixed mass of a general viscous compressible heat conducting capillary fluid bounded by a free boundary, Appl. Math. (Warsaw) 25 (1999), 489-511.

[9] E. Zadrzyńska and W. M. Zajączkowski, Local existence of solutions of a free boundary problem for equations of compressible viscous heat conducting capillary fluids, J. Appl. Anal., to appear.

[10] W. M. Zajączkowski, On nonstationary motion of a compressible barotropic viscous capillary fluid bounded by a free surface, SIAM J. Math. Anal. 25 (1994), 1-84.

Ewa Zadrzyńska

Institute of Mathematics

Polish Academy of Sciences

Śniadeckich 8

00-950 Warszawa, Poland

E-mail: emzad@impan.gov.pl

Department of Mathematics

and Information Science

Warsaw University of Technology

Plac Politechniki 1

00-661 Warszawa, Poland
Wojciech M. Zajączkowski Institute of Mathematics Polish Academy of Sciences Śniadeckich 8 00-950 Warszawa, Poland E-mail: wz@impan.gov.pl

Institute of Mathematics and Operations Research Military University of Technology Kaliskiego 2 00-908 Warszawa, Poland

Received on 14.7.1999;

revised version on 31.7.2000 